\title{
Betti numbers of random real hypersurfaces and determinants of random symmetric matrices
}

\author{
Damien Gayet, Jean-Yves Welschinger
}

May 31, 2018

\begin{abstract}
We asymptotically estimate from above the expected Betti numbers of random real hypersurfaces in smooth real projective manifolds. Our upper bounds grow as the square root of the degree of the hypersurfaces as the latter grows to infinity, with a coefficient involving the Kählerian volume of the real locus of the manifold as well as the expected determinant of random real symmetric matrices of given index. In particular, for large dimensions, these coefficients get exponentially small away from mid-dimensional Betti numbers. In order to get these results, we first establish the equidistribution of the critical points of a given Morse function restricted to the random real hypersurfaces.
\end{abstract}

MATHEMATICS SUBJECT ClASSIFICATION 2010: 14P25, 32U40, 60F10, 60B20

KEYWORDS: Real projective manifold, ample line bundle, random matrix, random polynomial

\section{Introduction}

How many real roots does a random real polynomial have? This question was answered by M. Kac in the 40's and, for a different measure, by E. Kostlan and M. Shub together with S. Smale in the 90's. In higher dimensions, this question may become: what is the topology of a random real hypersurface in a given smooth real projective manifold? The mean Euler characteristics of such random real hypersurfaces in $\mathbb{R} P^{n}$ has been computed by S. S. Podkorytov [19] and P. Bürgisser [4, while the mean total Betti number has been recently estimated from above by the authors [12] (see also [11]). In the case of spherical harmonics in dimension two, rather precise estimations have been obtained by F. Nazarov and M. Sodin [18.

Our aim is to improve our previous results [12] by getting upper bounds for all individual Betti numbers of random real hypersurfaces. Let $X$ be a smooth $n$-dimensional complex projective manifold defined over the reals and let $\mathbb{R} X$ be its real locus. Let $L$ be a real ample line bundle over $X$. We equip $L$ with a real Hermitian metric $h$ of positive curvature $\omega$ and $X$ with a normalized volume form $d x$. These induce an inner $L^{2}$-product on all spaces of global holomorphic real sections $\mathbb{R} H^{0}\left(X, L^{d}\right)$ for all tensor powers $L^{d}$ of $L, d>0$, see $\$ 1.1$. The latter spaces then inherit Gaussian probability measures $\mu_{\mathbb{R}}$, with respect to which we are going to consider random sections, see 
$\S 3.1 .1$ of [12] for a discussion on this choice (previously considered in [14, [21, [19], [4]) and on other possible ones (compare [20]).

For every generic section $\sigma \in \mathbb{R} H^{0}\left(X, L^{d}\right)$, the real locus $\mathbb{R} C_{\sigma}$ of its vanishing locus is a smooth hypersurface of $\mathbb{R} X$, if non empty. For every $i \in\{0, \cdots, n-1\}$, we denote by $b_{i}\left(\mathbb{R} C_{\sigma}\right)$ the infinimum over all Morse functions $f$ on $\mathbb{R} C_{\sigma}$ of the number of critical points of index $i$ of $f$. This fake Betti number bounds from above all $i$ th Betti numbers of $\mathbb{R} C_{\sigma}$, whatever the coefficient rings are, as follows from Morse theory (see, e.g., [17]). We then denote by $E\left(b_{i}\right)$ the average value of this fake Betti number, namely $E\left(b_{i}\right)=\int_{\mathbb{R} H^{0}\left(X, L^{d}\right)} b_{i}\left(\mathbb{R} C_{\sigma}\right) d \mu_{\mathbb{R}}(\sigma)$. Our aim is to prove the following upper bound for this expectation (see Corollary 2):

Theorem 1 Let $X$ be a smooth real projective manifold of dimension $n>0$ and $(L, h)$ be a real Hermitian line bundle of positive curvature $\omega$ over $X$. Then

$$
\limsup _{d \rightarrow \infty} \frac{1}{\sqrt{d}^{n}} E\left(b_{i}\right) \leq \frac{1}{\sqrt{\pi}} e_{\mathbb{R}}(i, n-1-i) \operatorname{Vol}_{h}(\mathbb{R} X) .
$$

Moreover, when $n=1$, the limsup is a limit and the inequality an equality, so that

$$
E\left(b_{0}\right) \underset{d \rightarrow \infty}{\sim} \frac{\text { Length }_{h}(\mathbb{R} X)}{\sqrt{\pi}} \sqrt{d} .
$$

In Theorem 1, $\operatorname{Vol}_{h}(\mathbb{R} X)$ denotes the Riemannian volume of $\mathbb{R} X$ for the Kähler metric induced by the curvature form $\omega$ of $h$. It turns out that Theorem 1, as well as Theorem 2 and Corollary 1, does not depend on the normalized volume form $d x$ chosen on $X$ to define the $L^{2}$-inner product on $\mathbb{R} H^{0}\left(X, L^{d}\right)$, compare [20].

The coefficient $e_{\mathbb{R}}(i, n-1-i)$ is itself a mathematical expectation, namely the average value of (the absolute value of) the determinant on symmetric matrices of signature $(i, n-1-i)$, see 1 1.1. More precisely, the space $\operatorname{Sym}(n-1, \mathbb{R})$ of square symmetric matrices of size $(n-1) \times(n-1)$ has a natural Gaussian measure that we also denote by $\mu_{\mathbb{R}}$. Let $\operatorname{Sym}(i, n-1-i, \mathbb{R})$ be the open subset of matrices of signature $(i, n-1-i)$. Then, for every $i \in\{0, \cdots, n-1\}$

$$
\begin{aligned}
e_{\mathbb{R}}(i, n-1-i) & =\int_{\operatorname{Sym}(i, n-1-i, \mathbb{R})}|\operatorname{det} A| d \mu_{\mathbb{R}}(A) \text { and } \\
e_{\mathbb{R}}(n-1) & =\int_{\operatorname{Sym}(n-1, \mathbb{R})}|\operatorname{det} A| d \mu_{\mathbb{R}}(A) .
\end{aligned}
$$

Here by convention, $e_{\mathbb{R}}(0)=e_{\mathbb{R}}(0,0)=1$.

Note that when $X=\mathbb{C} P^{1}, L=\mathcal{O}_{\mathbb{C} P^{1}}(1)$ and $h$ is the Fubini-Study metric, $\operatorname{Vol}_{F S}\left(\mathbb{R} P^{1}\right)=\sqrt{\pi}$, so that Theorem 1 recovers asymptotically the results of Kostlan and Shub-Smale, up to which a random degree $d$ real polynomial in one variable has $\sqrt{d}$ roots for our choice of the probability measure. The initial result by M. Kac was rather expecting $\frac{2}{\pi} \log d$ real roots, but for a different probability measure, see $\S 3.1 .1$ of [12. When $X=\mathbb{C} P^{2}$, P. Sarnak and I. Wigman informed us in 2011 that they were also able to bound $E\left(b_{0}\right)$ from above by a $O(d)$ term as in Theorem 1 . This Theorem 11 improves our previous results of [12, where the best upper bounds we could get were by $O\left(\sqrt{d \log d^{n}}\right)$ in some cases.

Theorem 1 turns out to be the consequence of a more precise equidistribution result. Namely, when $n>1$, we equip $\mathbb{R} X$ with a fixed Morse function $p: \mathbb{R} X \rightarrow \mathbb{R}$. 
Then, for every generic section $\sigma \in \mathbb{R} H^{0}\left(X, L^{d}\right), p$ restricts to a Morse function on $\mathbb{R} C_{\sigma}$ and we denote by $\operatorname{Crit}_{i}\left(p_{\mid \mathbb{R} C_{\sigma}}\right)$ the set of critical points of index $i$ of this restriction. We set

$$
\nu_{i}\left(\mathbb{R} C_{\sigma}\right)=\frac{1}{\sqrt{d}^{n}} \sum_{x \in C r i t_{i}\left(p_{\mathbb{R} C_{\sigma}}\right)} \delta_{x}
$$

the empirical measure on these critical points, where $\delta_{x}$ denotes the Dirac measure at $x$ and $E\left(\nu_{i}\right)=\int_{\mathbb{R} H^{0}\left(X, L^{d}\right)} \nu_{\sigma} d \mu_{\mathbb{R}}(\sigma)$. When $n=1, \nu_{0}$ denotes the empirical measure on $\mathbb{R} C_{\sigma}$. Then, we get (see Theorem 6)

Theorem 2 Under the hypotheses of Theorem 1, the measure $E\left(\nu_{i}\right)$ weakly converges to $\frac{1}{\sqrt{\pi}} e_{\mathbb{R}}(i, n-1-i) d_{\text {vol }}$ on $\mathbb{R} X$ as d grows to infinity.

In Theorem 2, $d v o l_{h}$ denotes the Lebesgue measure of $\mathbb{R} X$ induced by its Riemannian metric, which is itself induced by the Kähler metric of $X$ defined by $\omega$. Note that we also establish such an equidistribution result for critical points of complex hypersurfaces, where the Morse function $p$ on $\mathbb{R} X$ is replaced by a Lefschetz pencil on $X$ and $\mathbb{R} H^{0}\left(X, L^{d}\right)$ by $H^{0}\left(X, L^{d}\right)$, see Theorem 5. Similar equidistribution results can be found in [8], [9], [15], [12] or also [2], [6].

In order to prove Theorem 2, we roughly follow the approach of [21]. We introduce the incidence variety $\Sigma_{i}=\left\{(\sigma, x) \in \mathbb{R} H^{0}\left(X, L^{d}\right) \times \mathbb{R} X \mid x \in \operatorname{Crit}_{i}\left(p_{\mid \mathbb{R} C_{\sigma}}\right)\right\}$ and express $E\left(\nu_{\sigma}\right)$ as the push-forward onto $\mathbb{R} X$ of the Gaussian measure $\mu_{\mathbb{R}}$ of $\mathbb{R} H^{0}\left(X, L^{d}\right)$ "pulled-back" on $\Sigma_{i}$. This push-forward measure is then computed asymptotically thanks to the coarea formula and peak sections of Hörmander. The latter indeed make it possible to compute pointwise the measure in terms of the 2-jets of sections, see $\$ 1$.

Now, what are the values of the expectations $e_{\mathbb{R}}(n), n>0$, and how do these distribute between the different $e_{\mathbb{R}}(i, n-i), 0 \leq i \leq n$ ? We devote the second paragraph to this question and get (see Proposition 6. Proposition 7 and Corollary 3):

Theorem 3 When $n$ is odd, $e_{\mathbb{R}}(n)=\frac{2 \sqrt{2}}{\pi} \Gamma\left(\frac{n+2}{2}\right)$, while when $n$ is even,

$$
e_{\mathbb{R}}(n)=(-1)^{m} \frac{n !}{m ! 2^{n}}+(-1)^{m-1} \frac{4 \sqrt{2} n !}{\sqrt{\pi} m ! 2^{n}} \sum_{k=0}^{m-1}(-1)^{k} \frac{\Gamma(k+3 / 2)}{k !} .
$$

In both cases, $e_{\mathbb{R}}(n)$ is equivalent to $\frac{2 \sqrt{2}}{\pi} \Gamma\left(\frac{n+2}{2}\right)$ as $n$ grows to infinity.

The odd case in Theorem 3 was known, see $\S 26.5$ of [16], but we could only find the even case in terms of hypergeometric functions in the literature, see [7]. It turns out that $e_{\mathbb{R}}(n)$ is transcendental for odd $n$ and algebraic in $\mathbb{Q}(\sqrt{2})$ for even $n$. We can now rewrite the bound deduced from Theorem 1 for the expected total Betti number $E\left(b_{*}\right)=\sum_{i=0}^{n-1} E\left(b_{i}\right)$ as follows (see Remark 2).

Corollary 1 Under the hypotheses of Theorem 1, for every even $n>0$,

$$
\limsup _{d \rightarrow \infty} \frac{1}{\sqrt{d}^{n}} E\left(b_{*}\right) \leq \frac{2 \sqrt{2}}{\pi}\left(\frac{\operatorname{Vol}_{h}(\mathbb{R} X)}{V o l_{F S}\left(\mathbb{R} P^{n}\right)}\right) .
$$

For odd $n$, this inequality holds asymptotically in $n$. 
In particular, for every even-dimensional projective space, the right-hand side in Corollary 1 turns out not to depend on the dimension of the space. Finally, we get the following exponential decay away from the mid-dimensional Betti numbers (see Proposition 4):

Theorem 4 For every $\alpha \in\left[0,1 / 2\left[\right.\right.$, there exists $c_{\alpha}>0$, such that

$$
\sum_{i=0}^{\lfloor\alpha n\rfloor} e_{\mathbb{R}}(i, n-i) \leq \exp \left(-c_{\alpha} n^{2}\right) .
$$

This concentration near matrices having as many positive as negative eigenvalues actually follows from the large deviations estimates near Wigner semi-circle law established in [3]. As a consequence of Theorem 4, for large values of $n$, the upper bound for the expected total Betti number of $\mathbb{R} C_{\sigma}$ given by the right hand side of Theorem 1 distributes between the different Betti numbers in such a way that it gets concentrated around the mid-dimensional ones and exponentially decreases away from them.

The first paragraph of this paper is devoted to Theorems 1 and 2 . A key role is played by Hörmander peak sections, see $\$ 1.3$. Note that we also prove along the same lines the complex analogue of Theorem 2, which is of independent interest. The second paragraph is devoted to Theorems 3 and 4 and the study of determinants of random symmetric matrices.

Aknowledgements. The research leading to these results has received funding from the European Community's Seventh Framework Progamme ([FP7/2007-2013] [FP7/2007-2011]) under grant agreement $\mathrm{n}^{\mathrm{o}}$ [258204].

\section{Contents}

1 Expected Betti numbers of random real hypersurfaces 5

1.1 Notations . . . . . . . . . . . . . . . . . 5

1.2 Statement of the results . . . . . . . . . . . . 7 G

1.3 Hörmander peak sections . . . . . . . . . . . . . . . . . 9

1.4 Incidence varieties and evaluation maps . . . . . . . . . . . . . 12

1.5 Proofs of Theorems 5 and $6 \ldots \ldots \ldots$

2 Expected determinant of random symmetric matrices 20

2.1 Large random real symmetric matrices . . . . . . . . . . . . . . . 20

2.1.1 The energy functional . . . . . . . . . . . . . 20

2.1.2 Measure concentration around matrices of vanishing signature 21

2.1.3 Volume of the orthogonal group . . . . . . . . . . . . . 22

2.2 Determinants of random symmetric matrices . . . . . . . . . . 24

2.2.1 Complex symmetric matrices . . . . . . . . . . . . . . 24

2.2.2 Real symmetric matrices of odd size . . . . . . . . . 25

2.2.3 Real symmetric matrices of even size . . . . . . . . . . . . 27

2.2.4 Values of $e(p, q)$ for $p+q \leq 3 \ldots \ldots$. . . . . . . . . . . 


\section{Expected Betti numbers of random real hypersurfaces}

\section{$1.1 \quad$ Notations}

Let $X$ be a smooth complex projective manifold of positive dimension $n$. When $X$ is defined over $\mathbb{R}$, we denote by $c_{X}: X \rightarrow X$ the associated antiholomorphic involution, called real structure, and by $\mathbb{R} X \subset X$ the real locus of the manifold, that is the fixed point set of $c_{X}$. Likewise, let $L$ be an ample holomorphic line bundle over $X$ equipped with a Hermitian metric $h$ of positive curvature. We denote by $\omega$ the curvature form of $h$, so that for every local non-vanishing holomorphic section $e$ of $L$ defined over an open subset $U$ of $X$,

$$
\omega_{\mid U}=\frac{1}{2 i \pi} \partial \bar{\partial} \log h(e, e) .
$$

We denote by $g=\omega(., J$.) the induced Kähler metric on $X$, where $J$ denotes the complex structure of $T X$.

When $X$ and $L$ are defined over $\mathbb{R}$, we denote by $c_{L}$ the associated real structure of $L$ and assume that $h$ is real, so that $\overline{c_{L}^{*} h}=h$. The restriction of $g$ to $\mathbb{R} X$ is a Riemannian metric and we denote by $\operatorname{Vol}_{h}(\mathbb{R} X)$ the total volume of $\mathbb{R} X$ for the associated Lebesgue measure $d v \mathrm{vl}_{h}$. Note that the volume of $X$ is independent of the metric $h$ and equals $\operatorname{Vol}(X)=\int_{X} \frac{\omega^{n}}{n !}=\frac{1}{n !} \int_{X} c_{1}(L)^{n}$. We denote by $d x=\frac{1}{\int_{X} \omega^{n}} \omega^{n}$ the normalized volume form of $X$, or any volume form on $X$ with total volume one.

For every $d>0$, we denote by $L^{d}$ the $d$-th tensor power of $L$ and by $h^{d}$ the induced Hermitian metric on $L^{d}$. We denote by $H^{0}\left(X, L^{d}\right)$ its complex vector space of global holomorphic sections and by $N_{d}$ the dimension of $H^{0}\left(X, L^{d}\right)$. We denote then by $\langle.,$.$\rangle the L^{2}$-Hermitian product on this vector space, defined by the relation

$$
\forall \sigma, \tau \in H^{0}\left(X, L^{d}\right),\langle\sigma, \tau\rangle=\int_{X} h^{d}(\sigma, \tau) d x
$$

The associated Gaussian measure is denoted by $\mu_{\mathbb{C}}$. It is defined, for every open subset $U$ of $H^{0}\left(X, L^{d}\right)$, by

$$
\mu_{\mathbb{C}}(U)=\frac{1}{\pi^{N_{d}}} \int_{U} e^{-\|\sigma\|^{2}} d \sigma
$$

where $d \sigma$ denotes the Lebesgue measure of $H^{0}\left(X, L^{d}\right)$. When $L$ is defined over $\mathbb{R}$, we denote by $\mathbb{R} H^{0}\left(X, L^{d}\right)$ the real vector space of real sections of $L^{d}$, made of sections $\sigma \in H^{0}\left(X, L^{d}\right)$ satisfying $c_{L} \circ \sigma \circ c_{X}=\sigma$. Its dimension equals $N_{d}$. The Hermitian $L^{2}$-product $\langle.,$.$\rangle restricts to a scalar product on \mathbb{R} H^{0}\left(X, L^{d}\right)$, which we also denote by $\langle.,$.$\rangle . The associated Gaussian measure is denoted by \mu_{\mathbb{R}}$ and defined for every open subset $\mathbb{R} U$ of $\mathbb{R} H^{0}\left(X, L^{d}\right)$ by

$$
\mu_{\mathbb{R}}(\mathbb{R} U)=\frac{1}{\sqrt{\pi}^{N_{d}}} \int_{\mathbb{R} U} e^{-\|\sigma\|^{2}} d \sigma .
$$

For every $d>0$, we denote by $\Delta^{d}$ (resp. $\mathbb{R} \Delta^{d}$ ) the discriminant hypersurface of $H^{0}\left(X, L^{d}\right)$ (resp. $\mathbb{R} H^{0}\left(X, L^{d}\right)$ ), that is the set of sections $\sigma \in H^{0}\left(X, L^{d}\right)$ (resp. $\left.\sigma \in \mathbb{R} H^{0}\left(X, L^{d}\right)\right)$ which do not vanish transversally. For every $\sigma \in H^{0}\left(X, L^{d}\right) \backslash\{0\}$, we denote by $C_{\sigma}$ (resp. $\mathbb{R} C_{\sigma}$ ) the vanishing locus of $\sigma$ in $X$ (resp. its real locus when 
$\left.\sigma \in \mathbb{R} H^{0}\left(X, L^{d}\right)\right)$. For every $\sigma \in H^{0}\left(X, L^{d}\right) \backslash \Delta^{d}, C_{\sigma}$ is then a smooth hypersurface of $X$. When $\sigma$ is real, $\mathbb{R} C_{\sigma}$ is of dimension $n-1$ when non empty and we denote, for $i \in\{0, \cdots, n-1\}$, by $b_{i}\left(\mathbb{R} C_{\sigma}\right)$ the minimum number of critical points of a Morse function on $\mathbb{R} C_{\sigma}$. From Morse theory we know that $b_{i}\left(\mathbb{R} C_{\sigma}\right)$ is bigger than any of its $i$-th Betti number, whatever the coefficient ring is. When $X$ is real (resp. complex) and $n>1$, we equip its real locus with a Morse function

$$
p: \mathbb{R} X \rightarrow \mathbb{R}
$$

(resp. with a Lefschetz pencil $p: X \rightarrow \mathbb{C} P^{1}$ ). We then denote, for every $d>0$, by $\mathbb{R} \Delta_{p}^{d}$ (resp. $\left.\Delta_{p}^{d}\right)$ the locus of sections $\sigma \in \mathbb{R} H^{0}\left(X, L^{d}\right)\left(\right.$ resp. $\left.\sigma \in H^{0}\left(X, L^{d}\right)\right)$ such that $\sigma \in \mathbb{R} \Delta^{d}$ (resp. $\Delta^{d}$ ) or the restriction of $p$ to $\mathbb{R} C_{\sigma}$ (resp. $C_{\sigma}$ ) is not Morse (resp. not a Lefschetz pencil). For every $\sigma \in H^{0}\left(X, L^{d}\right) \backslash \Delta_{p}^{d}$, we denote by $\operatorname{Crit}\left(p_{\mid C_{\sigma}}\right)$ the set of critical points of the restriction of $p$ to $C_{\sigma}$ and set

$$
\nu\left(C_{\sigma}\right)=\frac{1}{d^{n}} \sum_{x \in C r i t\left(p_{\mid C_{\sigma}}\right)} \delta_{x}
$$

where $\delta_{x}$ denotes the Dirac measure of $X$ at the point $x$. When $\sigma$ is real, we denote similarly, for every $i \in\{0, \cdots n-1\}$, by $\operatorname{Crit}_{i}\left(p_{\mid \mathbb{R} C_{\sigma}}\right)$ the set of critical points of index $i$ of $p_{\mid \mathbb{R} C_{\sigma}}$, and set

$$
\nu_{i}\left(\mathbb{R} C_{\sigma}\right)=\frac{1}{\sqrt{d}^{n}} \sum_{x \in C r i t_{i}\left(p_{\mid \mathbb{R} C_{\sigma}}\right)} \delta_{x} .
$$

When $n=1$, we set likewise $\nu\left(C_{\sigma}\right)=\frac{1}{d} \sum_{x \in C_{\sigma}} \delta_{x}$ and when $\sigma \in \mathbb{R} H^{0}\left(X, L^{d}\right) \backslash \mathbb{R} \Delta^{d}$,

$$
\nu_{0}\left(\mathbb{R} C_{\sigma}\right)=\frac{1}{\sqrt{d}} \sum_{x \in \mathbb{R} C_{\sigma}} \delta_{x}
$$

For every $n \in \mathbb{N}^{*}$, denote by $\operatorname{Sym}(n, \mathbb{R})$ (resp. by $\operatorname{Sym}(n, \mathbb{C})$ ) the real (resp. complex) vector space of real (resp. complex) symmetric matrices of size $n \times n$. These vector spaces are of dimension $\frac{n(n+1)}{2}$ and we equip them with the basis $B$ given by the vectors $\widetilde{E}_{i i}=\sqrt{2} E_{i i}$ and $\widetilde{E}_{i j}=E_{i j}+E_{j i}, 1 \leq i<j \leq n$, where for every $1 \leq k, l \leq n, E_{k l}$ denotes the elementary matrix whose entry at the $i$-th row and $j$-th column equals 1 if $(i, j)=(k, l)$ and 0 otherwise. We equip then $\operatorname{Sym}(n, \mathbb{R})$ (resp. $\operatorname{Sym}(n, \mathbb{C}))$ with the scalar (resp. Hermitian) product turning $B$ into an orthonormal basis and we denote by $\|$.$\| the associated norm. We then denote by \mu_{\mathbb{R}}$ (resp. $\mu_{\mathbb{C}}$ ) the associated Gaussian probability measure, so that for every open subset $U$ of $\operatorname{Sym}(n, \mathbb{R})(\operatorname{resp} . V$ of $\operatorname{Sym}(n, \mathbb{C}))$,

$$
\mu_{\mathbb{R}}(U)=\frac{1}{\sqrt{\pi^{\frac{n(n+1)}{2}}}} \int_{U} e^{-\|A\|^{2}} d A \text { and } \mu_{\mathbb{C}}(V)=\frac{1}{\pi^{\frac{n(n+1)}{2}}} \int_{V} e^{-\|A\|^{2}} d A,
$$

where $d A$ denotes the Lebesgue measure. For every $p, q \in \mathbb{N}$, we denote by $\operatorname{Sym}(p, q, \mathbb{R})$ the open subset of $\operatorname{Sym}(p+q, \mathbb{R})$ made of non-degenerated matrices of signature $(p, q)$. 
We then set, for every integers $p, q, n$,

$$
\begin{aligned}
e_{\mathbb{C}}(n) & =E_{\mathbb{C}}\left(|\operatorname{det}|^{2}\right)=\int_{\operatorname{Sym}(n, \mathbb{C})}|\operatorname{det} A|^{2} d \mu_{\mathbb{C}}(A), \\
e_{\mathbb{R}}(n) & =E_{\mathbb{R}}(|\operatorname{det}|)=\int_{\operatorname{Sym}(n, \mathbb{R})}|\operatorname{det} A| d \mu_{\mathbb{R}}(A) \text { and } \\
e_{\mathbb{R}}(p, q) & =\int_{\operatorname{Sym}(p, q, \mathbb{R})}|\operatorname{det} A| d \mu_{\mathbb{R}}(A),
\end{aligned}
$$

so that $\sum_{\substack{p, q \in \mathbb{N} \\ p+q=n}} e_{\mathbb{R}}(p, q)=e_{\mathbb{R}}(n)$. By convention, $e_{\mathbb{R}}(0)=e_{\mathbb{C}}(0)=e_{\mathbb{R}}(0,0)=1$.

\subsection{Statement of the results}

Using the notations of $\$ 1.1$, we set, for every $d>0$,

$$
E(\nu)=\int_{H^{0}\left(X, L^{d}\right) \backslash \Delta_{p}^{d}} \nu\left(C_{\sigma}\right) d \mu_{\mathbb{C}}(\sigma)
$$

the average of the measure $\nu$.

Theorem 5 Let $X$ be a smooth complex projective manifold of dimension $n>0$ and $L$ be an ample holomorphic line bundle on $X$ equipped with a Hermitian metric of positive curvature $\omega$. Let $p: X \rightarrow \mathbb{C} P^{1}$ be a Lefschetz pencil. Then, the measure $E(\nu)$ weakly converges to $e_{\mathbb{C}}(n-1) \frac{\omega^{n}}{n !}=\omega^{n}$ as d grows to infinity.

The form $\frac{\omega^{n}}{n !}$ in Theorem 5 is the Kähler volume form defined by $\omega$. The equality $e_{\mathbb{C}}(n-1)\left(\frac{\omega^{n}}{n !}\right)=\omega^{n}$ follows from Proposition 5, see $\$ 2.2 .1$. By "weak convergence" in Theorem 5 we mean that for every continuous function $\chi: X \rightarrow \mathbb{R},\langle E(\nu), \chi\rangle$ converges to $\int_{X} \chi \omega^{n}$ as $d$ grows to infinity, where

$$
\langle E(\nu), \chi\rangle=\frac{1}{d^{n}} \int_{H^{0}\left(X, L^{d}\right) \backslash \Delta_{p}^{d}}\left(\sum_{x \in C r i t\left(p_{\mid C_{\sigma}}\right)} \chi(x)\right) d \mu_{\mathbb{C}}(x) .
$$

Note that Theorem 5 slightly improves Theorem 3 of [12]. Note also that this result, as well as the following Theorem 6 and Corollary 2 , does not depend on the normalized volume form $d x$ chosen on $X$ in order to define the $L^{2}$-inner product on $H^{0}\left(X, L^{d}\right)$, see \$1.1. Likewise, when $X$ and $L$ are real, we set for every $d>0$ and $i \in\{0, \cdots, n-1\}$,

$$
E\left(\nu_{i}\right)=\int_{\mathbb{R} H^{0}\left(X, L^{d}\right) \backslash \mathbb{R} \Delta_{p}^{d}} \nu_{i}\left(\mathbb{R} C_{\sigma}\right) d \mu_{\mathbb{R}}(\sigma)
$$

the average of the measure $\nu_{i}$.

Theorem 6 Let $X$ be a smooth real projective manifold of dimension $n>0$ and $L$ be a real ample holomorphic line bundle over $X$ equipped with a real Hermitian metric of positive curvature $\omega$. Let $p: \mathbb{R} X \rightarrow \mathbb{R}$ be a Morse function. Then, for every $i \in\{0, \cdots, n-1\}$, the measure $E\left(\nu_{i}\right)$ weakly converges to $\frac{1}{\sqrt{\pi}} e_{\mathbb{R}}(i, n-1-i) d v l_{h}$ as $d$ grows to infinity. 
Recall that $d v o l_{h}$ denotes the Lebesgue measure associated to the Riemannian metric on $\mathbb{R} X$ induced by the Kähler metric $g$ defined by $\omega$. Under the hypotheses of Theorem 6 and using the notations of $\$ 1.1$, we denote by

$$
E\left(b_{i}\right)=\int_{\mathbb{R} H^{0}\left(X, L^{d}\right) \backslash \mathbb{R} \Delta_{p}^{d}} b_{i}\left(\mathbb{R} C_{\sigma}\right) d \mu_{\mathbb{R}}(\sigma)
$$

the average value of the $i$-th Betti number.

Corollary 2 Under the hypotheses of Theorem 6 ,

$$
\limsup _{d \rightarrow \infty} \frac{1}{\sqrt{d}^{n}} E\left(b_{i}\right) \leq \frac{1}{\sqrt{\pi}} e_{\mathbb{R}}(i, n-1-i) \operatorname{Vol}_{h}(\mathbb{R} X)
$$

Moreover, when $n=1$, the $\lim \sup$ is a limit and the inequality an equality, so that

$$
E\left(b_{0}\right) \underset{d \rightarrow \infty}{\sim} \frac{\text { Length }_{h}(\mathbb{R} X)}{\sqrt{\pi}} \sqrt{d}
$$

Note that this Corollary 2 substantially improves Theorem 4 of [12].

Proof. By definition, for every $\sigma \in \mathbb{R} H^{0}\left(X, L^{d}\right) \backslash \mathbb{R} \Delta_{p}^{d}$,

$$
\frac{1}{\sqrt{d}^{n}} b_{i}\left(\mathbb{R} C_{\sigma}\right) \leq \int_{\mathbb{R} X} \nu_{i}\left(\mathbb{R} C_{\sigma}\right)
$$

with equality when $n=1$ (and $i=0$ ). By integration over $\mathbb{R} H^{0}\left(X, L^{d}\right) \backslash \mathbb{R} \Delta_{p}^{d}$, we deduce that

$$
\frac{1}{\sqrt{d}^{n}} E\left(b_{i}\right) \leq \int_{\mathbb{R} X} E\left(\nu_{i}\right),
$$

with equality when $n=1$. When $n=1$, the result follows from Theorem 6 and from the definition of $\operatorname{Vol}_{h}(\mathbb{R} X)=\int_{\mathbb{R} X} d v o l_{h}$. In general, we know from Theorem 6 that for every $\epsilon>0$, there exists $d_{0}>0$ such that

$$
\forall d>d_{0}, \int_{\mathbb{R} X} E\left(\nu_{i}\right) \leq \epsilon+\frac{1}{\sqrt{\pi}} e_{\mathbb{R}}(i, n-1-i) \operatorname{Vol}_{h}(\mathbb{R} X) .
$$

We get the result by taking the limsup on the left and having $\epsilon$ converge to zero.

Remark 1 When $X$ is the Riemann sphere $\mathbb{C} P^{1}, L=\mathcal{O}_{\mathbb{C} P^{1}}(1)$ and $h$ is the FubiniStudy metric, $X$ equipped with its Kählerian metric is isometric to the round sphere of radius $\frac{1}{2 \sqrt{\pi}}$ in the Euclidian three-space, so that its volume equals 1. It follows that $V_{\text {ol }}(\mathbb{R} X)=\sqrt{\pi}$, and Corollary 圆 then writes $E\left(b_{0}\right) \underset{d \rightarrow \infty}{\sim} \sqrt{d}$, which is consistent with Kostlan and Shub-Smale's results, see [14] and [21].

Remark 2 When $X=\mathbb{C} P^{n}, L=\mathcal{O}_{\mathbb{C} P^{n}}(1)$ and $h$ is the Fubini-Study metric, the geodesics $\mathbb{R} P^{1}$ of $\mathbb{R} P^{n}$ have length $\sqrt{\pi}$, so that $\mathbb{R} P^{n}$ is isometric to the quotient of the sphere of radius $\frac{1}{\sqrt{\pi}}$ by the antipodal map. Hence,

$$
\operatorname{Vol}_{F S}\left(\mathbb{R} P^{n}\right)=\frac{1}{2 \sqrt{\pi}^{n}} \operatorname{Vol}\left(S^{n}\right)=\frac{\sqrt{\pi}}{\Gamma\left(\frac{n+1}{2}\right)},
$$

where $S^{n}$ denotes the unit sphere in $\mathbb{R}^{n+1}$. 


\subsection{Hörmander peak sections}

With the notations of 91.1 , let $x$ be a point of $X$ (resp. $\mathbb{R} X)$. In a neighborhood of $x$ in $X$ there exists a local holomorphic (resp. real holomorphic) trivialization $e$ of $L$ whose associated potential $\Phi=-\log h(e, e)$ vanishes at $x$, where it reaches a local minimum with Hessian of type $(1,1)$. Let $\left(x_{1}, \cdots, x_{n}\right)$ be holomorphic (resp. real holomorphic) coordinates in the neighbourhood of $x=(0, \cdots, 0)$ in $X$, such that $\left(\frac{\partial}{\partial x_{1}}, \cdots, \frac{\partial}{\partial x_{n}}\right)$ be orthonormal at $x$ for the Kähler metric $g$. In these coordinates, the Taylor expansion of $\Phi$ writes:

$$
\Phi(y)=-\frac{1}{2 i} \partial \bar{\partial} \Phi(y, i y)+o\left(\|y\|^{2}\right)=\pi\|y\|^{2}+o\left(\|y\|^{2}\right)
$$

where the norm is induced by the Kähler metric $g$ at the point $x$.

The $L^{2}$-estimates of Hörmander make it possible, for every $d>0$, and after a small perturbation of $e^{d}$ in $L^{2}$-norm, to extend $e^{d}$ into a global holomorphic (resp. real holomorphic) section of $L^{d}$. The latter is called a Hörmander peak section. Moreover, G. Tian (Lemma 1.2 in [23]) showed that this procedure can be controlled up to every order, as long as $d$ be large enough. We recall this result in the following Lemma 1 where for every $r>0, B(x, r)$ denotes the ball centered at $x$ and of radius $r$ in $X$.

Lemma 1 (See [23], Lemma 1.2) Let $(L, h)$ be a holomorphic Hermitian line bundle of positive curvature $\omega$ over a smooth complex projective manifold $X$. Let $x \in X$, $\left(p_{1}, \cdots, p_{n}\right) \in \mathbb{N}^{n}$ and $p^{\prime}>p_{1}+\cdots+p_{n}$. There exists $d_{0} \in \mathbb{N}$ such that for every $d>d_{0}$, the bundle $L^{d}$ has a global holomorphic section $\sigma$ satisfying $\int_{X} h^{d}(\sigma, \sigma) d x=1$ for the volume form $d x=\frac{1}{\int_{X} \omega^{n}} \omega^{n}$ and

$$
\int_{X \backslash B\left(x, \frac{\log d}{\sqrt{d}}\right)} h^{d}(\sigma, \sigma) d x=O\left(\frac{1}{d^{2 p^{\prime}}}\right) .
$$

Moreover, if $\left(x_{1}, \cdots, x_{n}\right)$ are local holomorphic coordinates in the neighborhood of $x$, we can assume that in a neighborhood of $x$,

$$
\sigma\left(x_{1}, \cdots, x_{n}\right)=\lambda\left(x_{1}^{p_{1}} \cdots x_{n}^{p_{n}}+O\left(|x|^{2 p^{\prime}}\right)\right) e^{d}\left(1+O\left(\frac{1}{d^{2 p^{\prime}}}\right)\right),
$$

where

$$
\lambda^{-2}=\int_{B\left(x, \frac{\log d}{\sqrt{d}}\right)}\left|x_{1}^{p_{1}} \cdots x_{n}^{p_{n}}\right|^{2} h^{d}\left(e^{d}, e^{d}\right) d x
$$

and $e$ is a local trivialization of $L$ whose potential $\Phi=-\log h(e, e)$ reaches a local minimum at $x$ with Hessian $\pi \omega(., i$.).

This Lemma 1 admits a real counterpart Lemma 2 which is obtained by averaging the peak sections with the real structure:

Lemma 2 Let $(L, h)$ be a real holomorphic Hermitian line bundle of positive curvature $\omega$ over a smooth real projective manifold $X$. Let $x \in \mathbb{R} X,\left(p_{1}, \cdots, p_{n}\right) \in \mathbb{N}^{n}$ and $p^{\prime}>p_{1}+\cdots+p_{n}$. There exists $d_{0} \in \mathbb{N}$ such that for every $d>d_{0}$, the bundle $L^{d}$ has 
a global real holomorphic section $\sigma$ satisfying $\int_{X} h^{d}(\sigma, \sigma) d x=1$ for the volume form $d x=\frac{1}{\int_{X} \omega^{n}} \omega^{n}$ and

$$
\int_{X \backslash B\left(x, \frac{\log d}{\sqrt{d}}\right)} h^{d}(\sigma, \sigma) d x=O\left(\frac{1}{d^{2 p^{\prime}}}\right) .
$$

Moreover, if $\left(x_{1}, \cdots, x_{n}\right)$ are local real holomorphic coordinates in the neighborhood of $x$ in $X$, we can assume that in a neighborhood of $x$ in $X$,

$$
\sigma\left(x_{1}, \cdots, x_{n}\right)=\lambda\left(x_{1}^{p_{1}} \cdots x_{n}^{p_{n}}+O\left(|x|^{2 p^{\prime}}\right)\right) e^{d}\left(1+O\left(\frac{1}{d^{2 p^{\prime}}}\right)\right),
$$

where

$$
\lambda^{-2}=\int_{B\left(x, \frac{\log d}{\sqrt{d}}\right)}\left|x_{1}^{p_{1}} \cdots x_{n}^{p_{n}}\right|^{2} h^{d}\left(e^{d}, e^{d}\right) d x
$$

and $e$ is a local real trivialization of $L$ whose potential $\Phi=-\log h(e, e)$ reaches a local minimum at $x$ with Hessian $\pi \omega(., i$.$) .$

Let $\sigma_{0}$ be a section given by Lemma 1 in coordinates $\left(x_{1}, \cdots, x_{n}\right)$ with $p^{\prime}=3$ and $p_{1}=\cdots=p_{n}=0$. Likewise, for every $j \in\{1, \cdots, n\}$, let $\sigma_{j}$ be a section given by Lemma 1 with $p^{\prime}=3, p_{j}=1$ and $p_{k}=0$ for $k \in\{1, \cdots, n\} \backslash\{j\}$. Finally, for every $1 \leq k \leq l \leq n$, let $\sigma_{k, l}$ be a section given by Lemma 1 with $p^{\prime}=3, p_{j}=0$ for every $j \in\{1, \cdots, n\} \backslash\{k, l\}$ and $p_{k}=p_{l}=1$ if $k \neq l$, while $p_{k}=2$ otherwise. All these sections have their norms concentrated in the neighbourhood of $x$ being close to 0 outside of a ball of radius $\frac{\log d}{\sqrt{d}}$ (from the mean value inequality, see Theorem 4.2.13 of [13] for instance). Likewise, by Lemma 1, the Taylor expansions of these sections write:

$$
\begin{aligned}
\sigma_{0}(y) & =\left(\lambda_{0}+O\left(\|y\|^{6}\right)\right) e^{d}(y)\left(1+O\left(\frac{1}{d^{6}}\right)\right) \\
\forall j \in\{1, \cdots, n\}, \sigma_{j}(y) & =\left(\lambda_{1} y_{j}+O\left(\|y\|^{6}\right)\right) e^{d}(y)\left(1+O\left(\frac{1}{d^{6}}\right)\right) \text { and } \\
\forall k, l \in\{1, \cdots, n\}, k \neq l, \sigma_{k, l}(y) & =\left(\lambda_{(1,1)} y_{k} y_{l}+O\left(\|y\|^{6}\right)\right) e^{d}(y)\left(1+O\left(\frac{1}{d^{6}}\right)\right), \text { whereas } \\
\forall k \in\{1, \cdots, n\}, \sigma_{k, k}(y) & =\left(\lambda_{(2,0)} y_{k}^{2}+O\left(\|y\|^{6}\right)\right) e^{d}(y)\left(1+O\left(\frac{1}{d^{6}}\right)\right) .
\end{aligned}
$$

The asymptotic values of the constants $\lambda_{0}, \lambda_{1}, \lambda_{(1,1)}$ and $\lambda_{(2,0)}$ are given in Lemma 3 (compare Lemma 2.1 of [23]):

Lemma 3 Under the hypotheses of Lemma 1, let $\delta_{L}=\int_{X} c_{1}(L)^{n}$ be the degree of $L$. Then

$$
\begin{array}{r}
\lim _{d \rightarrow \infty} \frac{1}{\sqrt{d}^{n}} \lambda_{0}=\sqrt{\delta_{L}} \quad ; \quad \lim _{d \rightarrow \infty} \frac{1}{\sqrt{d}^{n+1}} \lambda_{1}=\sqrt{\pi} \sqrt{\delta_{L}} \\
\lim _{d \rightarrow \infty} \frac{1}{\sqrt{d}^{n+2}} \lambda_{(1,1)}=\pi \sqrt{\delta_{L}} \quad \text { and } \quad \lim _{d \rightarrow \infty} \frac{1}{\sqrt{d}^{n+2}} \lambda_{(2,0)}=\frac{\pi}{\sqrt{2}} \sqrt{\delta_{L}},
\end{array}
$$

for the inner $L^{2}$-product induced by the volume form $d x=\frac{1}{\int_{X} \omega^{n}} \omega^{n}$. 
These values differ from the ones given in Lemma 2.1 of [23] by a constant $\pi^{n}$ since our choice of the Kähler metric slightly differs from [23].

Proof. From Lemma 1, $\lambda_{0}^{-2}$ is equivalent, as $d$ grows to infinity, to

$$
\frac{1}{\int_{X} \omega^{n}} \int_{\mathbb{C}^{n}} e^{-d \pi\|y\|^{2}} d \operatorname{vol}(y)=\frac{1}{\int_{X} d^{n} \omega^{n} \pi^{n}} \int_{\mathbb{C}^{n}} e^{-\|z\|^{2}} d \operatorname{vol}(z)=\frac{1}{\int_{X} d^{n} \omega^{n}},
$$

so that $\lambda_{0} \underset{d \rightarrow \infty}{\sim} \sqrt{\int_{X} c_{1}(L)^{n} d^{n}}$. Likewise, $\lambda_{1}^{-2}$ is equivalent to

$$
\frac{1}{\int_{X} \omega^{n}} \int_{\mathbb{C}^{n}}\left|y_{1}\right|^{2} e^{-d \pi\|y\|^{2}} d \operatorname{vol}(y)=\frac{1}{d \pi \int_{X} d^{n} \omega^{n} \pi^{n}} \int_{\mathbb{C}^{n}}\left|z_{1}\right|^{2} e^{-\|z\|^{2}} d \operatorname{vol}(z)=\frac{1}{d \pi \int_{X} d^{n} \omega^{n}},
$$

so that $\lambda_{1} \underset{d \rightarrow \infty}{\sim} \sqrt{d \pi} \lambda_{0}$. We obtain in the same way $\lambda_{(1,1)} \underset{d \rightarrow \infty}{\sim} d \pi \lambda_{0}$, whereas $\lambda_{(2,0)}^{-1}$ is equivalent to

$$
\frac{1}{\int_{X} \omega^{n}} \int_{\mathbb{C}^{n}}\left|y_{1}\right|^{4} e^{-d \pi\|y\|^{2}} d \operatorname{vol}(y)=\frac{1}{(d \pi)^{2} \int_{X} d^{n} \omega^{n} \pi^{n}} \int_{\mathbb{C}^{n}}\left|z_{1}\right|^{4} e^{-\|z\|^{2}}=\frac{2}{(d \pi)^{2} \int_{X} d^{n} \omega^{n}} .
$$

Hence, $\lambda_{(2,0)} \underset{d \rightarrow \infty}{\sim} \frac{d \pi}{\sqrt{2}} \lambda_{0}$.

Let $\nabla^{X}$ be a torsion-free connection on $T X$ and $\nabla^{L}$ be a connection (resp. real) on $L$. The connections $\nabla^{X}$ and $\nabla^{L}$ induce a connection denoted by $\nabla^{X, L}$ on $T^{*} X \otimes L$. We then set

$$
\nabla^{2} \sigma=\nabla^{X, L}\left(\nabla^{L} \sigma\right) \in \operatorname{End}\left(T X, T^{*} X \otimes L^{d}\right) .
$$

Now, the sections $\left(\sigma_{i}\right)_{0 \leq i \leq n}$ and $\left(\sigma_{k, l}\right)_{1 \leq k \leq l \leq n}$ define a basis of a complement of the subspace of sections of $H^{0}\left(X, L^{d}\right)\left(\right.$ resp. $\left.\mathbb{R} H^{0}\left(X, L^{d}\right)\right)$ whose 2 -jets at $x$ vanish, which is denoted by

$$
\begin{aligned}
H_{3 x} & =\left\{\sigma \in H^{0}\left(X, L^{d}\right) \mid \sigma(x)=0, \nabla^{L} \sigma_{\mid x}=0 \text { and } \nabla^{2} \sigma_{\mid x}=0\right\} \\
\text { (resp. } \mathbb{R} H_{3 x} & \left.=\left\{\sigma \in \mathbb{R} H^{0}\left(X, L^{d}\right) \mid \sigma(x)=0, \nabla^{L} \sigma_{\mid x}=0 \text { and } \nabla^{2} \sigma_{\mid x}=0\right\}\right) .
\end{aligned}
$$

This basis is not orthonormal and its spanned subspace is not orthogonal to $H_{3 x}$. However, from Lemma 3.1 of [23], we know that it becomes closer and closer to being orthonormal as $d$ grows to infinity, see Lemma 4 , as long as the chosen volume form is $d x=\frac{1}{\int_{X} \omega^{n}} \omega^{n}$, see Remark 3 ,

Lemma 4 (See [23], Lemma 3.1) The sections $\left(\sigma_{i}\right)_{0 \leq i \leq n}$ and $\left(\sigma_{k, l}\right)_{1 \leq k \leq l \leq n}$ have $L^{2}$-norm equal to one and their pairwise scalar product are $O\left(\frac{1}{d}\right)$. Likewise, their scalar products with every unitary element of $H_{3 x}$ are $O\left(\frac{1}{d^{3 / 2}}\right)$.

Remark 3 If the $L^{2}$-scalar product is induced by a volume form different from $d x=$ $\frac{1}{\int_{X} \omega^{n}} \omega^{n}$, say $d x=f(x) \frac{1}{\int_{X} \omega^{n}} \omega^{n}$, then Lemma 4 remains unchanged except that the $L^{2}$-norms of the sections, instead of being one, would converge to $\sqrt{f(x)}$ from (1). 


\subsection{Incidence varieties and evaluation maps}

Using the notations of $\$ 1.1$, let us denote by $\operatorname{Crit}(p)\left(\operatorname{resp} . \operatorname{Crit}_{i}(p)\right)$ the finite set of critical points of $p$ (resp. of index $i$ ) and by Base $(p)$ its base locus. Under the hypotheses of Theorem 5 (resp. Theorem 6) and following [21, we set

$$
\Sigma=\left\{(\sigma, x) \in\left(H^{0}\left(X, L^{d}\right) \backslash \Delta_{p}^{d}\right) \times\left(X \backslash(\operatorname{Crit}(p) \cup \operatorname{Base}(p)) \mid x \in \operatorname{Crit}\left(p_{\mid C_{\sigma}}\right)\right\},\right.
$$

$\left(\operatorname{resp} . \Sigma_{i}=\left\{(\sigma, x) \in\left(\mathbb{R} H^{0}\left(X, L^{d}\right) \backslash \mathbb{R} \Delta_{p}^{d}\right) \times(\mathbb{R} X \backslash \operatorname{Crit}(p)) \mid x \in \operatorname{Crit}_{i}\left(p_{\mid \mathbb{R} C_{\sigma}}\right)\right\}\right)$,

and

$$
\begin{aligned}
\pi_{1}:(\sigma, x) \in \Sigma & \mapsto \sigma \in H^{0}\left(X, L^{d}\right) \text { and } \pi_{2}:(\sigma, x) \in \Sigma \mapsto x \in X \\
\text { (resp. } \pi_{1}:(\sigma, x) \in \Sigma_{i} & \left.\mapsto \sigma \in \mathbb{R} H^{0}\left(X, L^{d}\right) \text { and } \pi_{2}:(\sigma, x) \in \Sigma_{i} \mapsto x \in \mathbb{R} X\right)
\end{aligned}
$$

the associated projections on these incidence varieties.

For every $\left(\sigma_{0}, x_{0}\right) \in \Sigma$ (resp. $\left.\left(\sigma_{0}, x_{0}\right) \in \Sigma_{i}\right)$, there exists a neighbourhood $U$ (resp. $\mathbb{R} U$ ) of $\sigma_{0}$ in $H^{0}\left(X, L^{d}\right)$ (resp. $\mathbb{R} H^{0}\left(X, L^{d}\right)$ ) and a neighbourhood $V$ (resp. $\mathbb{R} V$ ) of $x_{0}$ in $X$ (resp. $\mathbb{R} X$ ) such that for every $\sigma \in U$ (resp. $\sigma \in \mathbb{R} U$ ), the function $p_{\mid C_{\sigma}}$ (resp. $p_{\mid \mathbb{R} C_{\sigma}}$ ) has a unique critical point (resp. critical point of index $i$ ) in $V$ (resp. $\mathbb{R} V)$. We deduce from this an evaluation map at the critical point

$$
\begin{aligned}
e v_{\left(\sigma_{0}, x_{0}\right)}: \sigma \in U & \mapsto x \in \operatorname{Crit}\left(p_{\mid C_{\sigma}}\right) \cap V \\
\left(\operatorname{resp} . e v_{\left(\sigma_{0}, x_{0}\right)}: \sigma \in \mathbb{R} U\right. & \left.\mapsto x \in \operatorname{Crit}\left(p_{\mid \mathbb{R} C_{\sigma}}\right) \cap \mathbb{R} V\right),
\end{aligned}
$$

so that $\Sigma \cap(U \times V)\left(\right.$ resp. $\left.\Sigma_{i} \cap(\mathbb{R} U \times \mathbb{R} V)\right)$ is the graph of $e v_{\left(\sigma_{0}, x_{0}\right)}$. This evaluation map is constant on $\pi_{1}\left(\pi_{2}^{-1}\left(x_{0}\right)\right) \cap U$, so that its differential $d_{\mid \sigma_{0}} e v_{\left(\sigma_{0}, x_{0}\right)}$ vanishes on $T_{\sigma_{0}} \pi_{1}\left(\pi_{2}^{-1}\left(x_{0}\right)\right) \simeq \pi_{1}\left(\pi_{2}^{-1}\left(x_{0}\right)\right)$. When $n=1$, we agree that $\pi_{1}\left(\pi_{2}^{-1}(x)\right)=\{\sigma \in$ $\left.H^{0}\left(X, L^{d}\right) \mid \sigma(x)=0\right\}$. We denote by $d_{\mid \sigma_{0}} e v_{\left(\sigma_{0}, x_{0}\right)}^{\perp}$ the restriction of $d_{\mid \sigma_{0}} e v_{\left(\sigma_{0}, x_{0}\right)}$ to the orthogonal complement of $\pi_{1}\left(\pi_{2}^{-1}\left(x_{0}\right)\right)$ in $H^{0}\left(X, L^{d}\right)\left(\operatorname{resp} . \mathbb{R} H^{0}\left(X, L^{d}\right)\right)$.

Proposition 1 Under the hypotheses of Theorem 5 (resp. Theorem 6),

$$
\begin{aligned}
E(\nu) & =\frac{1}{d^{n}}\left(\pi_{2}\right)_{*}\left(\pi_{1}^{*} d \mu_{\mathbb{C}}\right) \\
\left(\text { resp. } E\left(\nu_{i}\right)\right. & \left.=\frac{1}{\sqrt{d}^{n}}\left(\pi_{2}\right)_{*}\left(\pi_{1}^{*} d \mu_{\mathbb{R}}\right)\right) .
\end{aligned}
$$

Moreover, at every point $x$ of $X \backslash(\operatorname{Crit}(p) \cup \operatorname{Base}(p))$ (resp. $\mathbb{R} X \backslash \operatorname{Crit}(p))$,

$$
\begin{aligned}
\left(\pi_{2}\right)_{*}\left(\pi_{1}^{*} d \mu_{\mathbb{C}}\right) & =\frac{1}{\pi^{n}}\left(\int_{\pi_{1}\left(\pi_{2}^{-1}(x)\right)}\left|\operatorname{det} d_{\mid \sigma} e v_{(\sigma, x)}^{\perp}\right|^{-2} d \mu_{\mathbb{C}}(\sigma)\right) \frac{\omega^{n}}{n !} \\
\left(\operatorname{resp} .\left(\pi_{2}\right)_{*}\left(\pi_{1}^{*} d \mu_{\mathbb{R}}\right)\right. & \left.=\frac{1}{\sqrt{\pi}^{n}}\left(\int_{\pi_{1}\left(\pi_{2}^{-1}(x)\right)}\left|\operatorname{det} d_{\mid \sigma} e v_{(\sigma, x)}^{\perp}\right|^{-1} d \mu_{\mathbb{R}}(\sigma)\right) d v o l_{h}\right) .
\end{aligned}
$$

Note that $\pi_{1}$ is a map between manifolds of the same dimension, while $\mu_{\mathbb{R}}$ and $\mu_{\mathbb{C}}$ are absolute values of volume forms, so that the pull-backs $\pi_{1}^{*} d \mu_{\mathbb{R}}$ and $\pi_{1}^{*} d \mu_{\mathbb{C}}$ are well defined. 
Remark 4 The pointwise expression of $\left(\pi_{2}\right)_{*}\left(\pi_{1}^{*} d \mu_{\mathbb{C}}\right)\left(\operatorname{resp} .\left(\pi_{2}\right)_{*}\left(\pi_{1}^{*} d \mu_{\mathbb{R}}\right)\right)$ is invariant under dilation of the $L^{2}$-inner product $\left\langle.\right.$, . . on $H^{0}\left(X, L^{d}\right)\left(\right.$ resp. on $\left.\mathbb{R} H^{0}\left(X, L^{d}\right)\right)$. Indeed, for every $\lambda \in \mathbb{C}$ (resp. $\lambda \in \mathbb{R}),\left(\sigma_{0}, x_{0}\right)$ in $\Sigma$ (resp. in $\left.\Sigma_{i}\right)$ and $\sigma$ in a neighborhood of $\sigma_{0}$, ev $v_{\left(\sigma_{0}, x_{0}\right)}(\sigma)=e v_{\left(\lambda \sigma_{0}, x_{0}\right)}(\lambda \sigma)$ so that $d_{\mid \sigma_{0}} e v_{\left(\sigma_{0}, x_{0}\right)}=\lambda d_{\mid \lambda \sigma_{0}} e v_{\left(\lambda \sigma_{0}, x_{0}\right)}$. We deduce that

$$
\operatorname{det} d_{\mid \sigma_{0}} e v_{\left(\sigma_{0}, x_{0}\right)}^{\perp}=\lambda^{n} \operatorname{det} d_{\mid \lambda \sigma_{0}} e v_{\left(\lambda \sigma_{0}, x_{0}\right)}^{\perp}
$$

if both determinants are computed in the orthonormal basis for the same inner product $\langle.,$.$\rangle at the source, but \operatorname{det} d_{\mid \sigma_{0}} e v_{\left(\sigma_{0}, x_{0}\right)}^{\perp}$ becomes equal to $\operatorname{det} d_{\mid \lambda \sigma_{0}} e v_{\left(\lambda \sigma_{0}, x_{0}\right)}^{\perp}$ when the latter is computed in an orthonormal basis for the inner product dilated by $\lambda^{2}$. Since under such a dilation the associated Gaussian measures are just push-forwards one with respect to the other by the corresponding homothety, the invariance follows.

Proof. Let $\chi: X \rightarrow \mathbb{R}$ be a continuous function. By definition,

$$
\begin{aligned}
\langle E(\nu), \chi\rangle & =\frac{1}{d^{n}} \int_{H^{0}\left(X, L^{d}\right) \backslash \Delta_{p}^{d}}\left(\sum_{x \in \operatorname{Crit}\left(p_{\mid C_{\sigma}}\right)} \chi(x)\right) d \mu_{\mathbb{C}}(\sigma) \\
& =\frac{1}{d^{n}} \int_{\Sigma}\left(\pi_{2}^{*} \chi\right)\left(\pi_{1}^{*} d \mu_{\mathbb{C}}\right) \\
& =\frac{1}{d^{n}} \int_{X} \chi\left(\pi_{2}\right)_{*}\left(\pi_{1}^{*} d \mu_{\mathbb{C}}\right) .
\end{aligned}
$$

But from the coarea formula (see Theorem 3.2.3 of [10] or Theorem 1 of [21]), for every $x \in X \backslash(\operatorname{Crit}(p) \cup \operatorname{Base}(p))$,

$$
\left(\pi_{2}\right)_{*}\left(\pi_{1}^{*} d \mu_{\mathbb{C}}\right)_{\mid x}=\frac{1}{\pi^{N_{d}}}\left(\int_{\pi_{1}\left(\pi_{2}^{-1}(x)\right)}\left|\operatorname{det} d_{\mid \sigma} e v_{(\sigma, x)}^{\perp}\right|^{-2} e^{-\|\sigma\|^{2}} d \sigma\right) \frac{\omega^{n}}{n !}
$$

since the Jacobian of $d_{\mid \sigma} e v_{(\sigma, x)}^{\perp}$, which is $\mathbb{C}$-linear and computed with respect to the volume forms $d \sigma$ at the source and $\frac{\omega^{n}}{n !}$ at the target, equals $\left|\operatorname{det} d_{\mid \sigma} e v_{(\sigma, x)}^{\perp}\right|^{2}$. We deduce that

$$
\left(\pi_{2}\right)_{*}\left(\pi_{1}^{*} d \mu_{\mathbb{C}}\right)_{\mid x}=\frac{1}{\pi^{n}}\left(\int_{\pi_{1}\left(\pi_{2}^{-1}(x)\right)}\left|\operatorname{det} d_{\mid \sigma} e v_{(\sigma, x)}^{\perp}\right|^{-2} d \mu_{\mathbb{C}}(\sigma)\right) \frac{\omega^{n}}{n !} . x .
$$

Under the hypotheses of Theorem $[$, if $\chi$ denotes now a continuous function $\chi: \mathbb{R} X \rightarrow$ $\mathbb{R}$, we obtain likewise

$$
\begin{aligned}
\left\langle E\left(\nu_{i}\right), \chi\right\rangle & =\frac{1}{\sqrt{d}^{n}} \int_{\Sigma_{i}}\left(\pi_{2}^{*} \chi\right)\left(\pi_{1}^{*} d \mu_{\mathbb{R}}\right) \\
& =\frac{1}{\sqrt{d}^{n}} \int_{\mathbb{R} X} \chi\left(\pi_{2}\right)_{*}\left(\pi_{1}^{*} d \mu_{\mathbb{R}}\right) .
\end{aligned}
$$

The coarea formula implies now for every $x \in \mathbb{R} X \backslash \operatorname{Crit}(p)$ the relation

$$
\left(\pi_{2}\right)_{*}\left(\pi_{1}^{*} d \mu_{\mathbb{R}}\right)_{\mid x}=\frac{1}{\sqrt{\pi}^{n}}\left(\int_{\pi_{1}\left(\pi_{2}^{-1}(x)\right)}\left|\operatorname{det} d_{\mid \sigma} e v_{(\sigma, x)}^{\perp}\right|^{-1} d \mu_{\mathbb{R}}(\sigma)\right) d v o l_{h \mid x}
$$


We are going to compute the Jacobian $\left|\operatorname{det} d_{\mid \sigma} e v_{(\sigma, x)}^{\perp}\right|$ appearing in Proposition 1. For every $x \in X \backslash(\operatorname{Crit}(p) \cup \operatorname{Base}(p))$ (resp. $x \in \mathbb{R} X \backslash$ Crit $(p))$, we denote by

$$
\begin{aligned}
K_{x} & =\operatorname{ker} d_{x} p \subset T_{x} X \\
\left(\operatorname{resp} . \mathbb{R} K_{x}\right. & \left.=\operatorname{ker} d_{x} p \subset T_{x} \mathbb{R} X\right)
\end{aligned}
$$

the kernel of $d_{x} p$ and set

$$
\begin{aligned}
H_{x} & =\left\{\sigma \in H^{0}\left(X, L^{d}\right) \mid \sigma(x)=0\right\} \\
\text { (resp. } \mathbb{R} H_{x} & \left.=\left\{\sigma \in \mathbb{R} H^{0}\left(X, L^{d}\right) \mid \sigma(x)=0\right\} .\right)
\end{aligned}
$$

We now assume that the torsion-free connection $\nabla^{X}$ preserves the distribution $K$ on $X \backslash(\operatorname{Crit}(p) \cup \operatorname{Base}(p))$. This means that for every local vector field $v$ of $X$ taking value in $K$, we assume that $\nabla^{X} v$ also gets values in $K$. For every $(\sigma, x) \in \Sigma$, we set

$$
\lambda_{(\sigma, x)}^{\prime}=\frac{\nabla^{L} \sigma}{\dot{\sigma}} \dot{\sigma} \in \operatorname{End}\left(T_{x} X / K_{x}, H^{0}\left(X, L^{d}\right) / H_{x}\right)
$$

where $\dot{\sigma}$ denotes any non-trivial element of $H^{0}\left(X, L^{d}\right) / H_{x}$. We consider $\nabla^{2} \sigma$ as a bilinear form on $K_{x}$, that is $\nabla^{2} \sigma \in \operatorname{End}\left(K_{x}, K_{x}^{*} \otimes L_{x}^{d}\right)$. Hence,

$$
\operatorname{det}\left(\nabla^{2} \sigma\right) \in \operatorname{End}\left(\wedge^{n-1} K_{x}, \wedge^{n-1} K_{x}^{*} \otimes L_{x}^{d(n-1)}\right) .
$$

Define also the bilinear form

$$
\nabla^{L}: \quad(v, \dot{\sigma}) \in K_{x} \times H_{x} / \pi_{1}\left(\pi_{2}^{-1}(x)\right) \mapsto \nabla_{v}^{L} \dot{\sigma} \in L_{x}^{d}
$$

which we consider as an element of $\operatorname{End}\left(K_{x},\left(H_{x} / \pi_{1}\left(\pi_{2}^{-1}(x)\right)\right)^{*} \otimes L_{x}^{d}\right)$ and denote abusively by $\nabla^{L}$. It follows that

$$
\operatorname{det}\left(\nabla^{L}\right) \in \operatorname{End}\left(\wedge^{n-1} K_{x}, \wedge^{n-1}\left(H_{x} / \pi_{1}\left(\pi_{2}^{-1}(x)\right)\right)^{*} \otimes L_{x}^{d(n-1)}\right)
$$

and we set

$$
\lambda_{(\sigma, x)}^{\prime \prime}=\frac{\operatorname{det} \nabla^{2} \sigma}{\operatorname{det}\left(\nabla^{L}\right)} \in \operatorname{End}\left(\wedge^{n-1} K_{x}, \wedge^{n-1}\left(H_{x} / \pi_{1}\left(\pi_{2}^{-1}(x)\right)\right)\right) .
$$

Finally, we set

$$
\lambda_{(\sigma, x)}=\lambda_{(\sigma, x)}^{\prime} \wedge \lambda_{(\sigma, x)}^{\prime \prime} \in \operatorname{End}\left(\wedge^{n} T_{x} X, \wedge^{n}\left(H^{0}\left(X, L^{d}\right) / \pi_{1}\left(\pi_{2}^{-1}(x)\right)\right)\right)
$$

when $n>1$ and $\lambda_{(\sigma, x)}=\lambda_{(\sigma, x)}^{\prime}$ when $n=1$.

In the real case, $\nabla^{X}$ denotes a torsion-free connection on $T \mathbb{R} X_{\mid \mathbb{R} X \backslash \operatorname{Crit}(p)}$ which preserves the distribution $\mathbb{R} K$, while $\nabla^{L}$ is real. For every $(\sigma, x) \in \Sigma_{i}, \lambda_{(\sigma, x)}^{\prime}$ belongs then to $\operatorname{End}\left(T_{x} \mathbb{R} X / \mathbb{R} K_{x}, \mathbb{R} H^{0}\left(X, L^{d}\right) / \mathbb{R} H_{x}\right)$ and $\nabla^{2} \sigma$ to $\operatorname{End}\left(\mathbb{R} K_{x}, \mathbb{R} K_{x}^{*} \otimes \mathbb{R} L_{x}^{d}\right)$, so that

$$
\operatorname{det}\left(\nabla^{2}\right) \in \operatorname{End}\left(\wedge^{n-1} \mathbb{R} K_{x}, \wedge^{n-1} \mathbb{R} K_{x}^{*} \otimes \mathbb{R} L_{x}^{d(n-1)}\right) .
$$

The bilinear form

$$
(v, \dot{\sigma}) \in \mathbb{R} K_{x} \times \mathbb{R} H_{x} / \pi_{1}\left(\pi_{2}^{-1}(x)\right) \mapsto \nabla_{v}^{L} \dot{\sigma} \in \mathbb{R} L_{x}^{d}
$$


is considered as an element of $\operatorname{End}\left(\mathbb{R} K_{x},\left(\mathbb{R} H_{x} / \pi_{1}\left(\pi_{2}^{-1}(x)\right)\right)^{*} \otimes \mathbb{R} L_{x}^{d}\right)$, so that

$$
\operatorname{det}\left(\nabla^{L}\right) \in \operatorname{End}\left(\wedge^{n-1} \mathbb{R} K_{x}, \wedge^{n-1}\left(\mathbb{R} H_{x} / \pi_{1}\left(\pi_{2}^{-1}(x)\right)\right)^{*} \otimes \mathbb{R} L_{x}^{d(n-1)}\right) .
$$

Finally,

$$
\begin{aligned}
\lambda_{(\sigma, x)}^{\prime \prime} & \in \operatorname{End}\left(\wedge^{n-1} \mathbb{R} K_{x}, \wedge^{n-1}\left(\mathbb{R} H_{x} / \pi_{1}\left(\pi_{2}^{-1}(x)\right)\right)\right) \\
\text { while } \lambda_{(\sigma, x)} & \in \operatorname{End}\left(\wedge^{n} T_{x} \mathbb{R} X, \wedge^{n}\left(\mathbb{R} H^{0}\left(X, L^{d}\right) / \pi_{1}\left(\pi_{2}^{-1}(x)\right)\right)\right)
\end{aligned}
$$

when $n>1$ and $\lambda_{(\sigma, x)}=\lambda_{(\sigma, x)}^{\prime}$ when $n=1$.

Proposition 2 Under the hypotheses of Theorem 5 (resp. Theorem [6), let $\left(\sigma_{0}, x_{0}\right) \in$ $\Sigma$ (resp. $\left.\left(\sigma_{0}, x_{0}\right) \in \Sigma_{i}\right)$. Then, $\operatorname{det}\left(d_{\mid \sigma_{0}} e v_{\left(\sigma_{0}, x_{0}\right)}^{\perp}\right)^{-1}=(-1)^{n} \lambda_{\left(\sigma_{0}, x_{0}\right)}$.

Proof. Consider neighbourhoods $U$ and $V$ of $\sigma_{0}$ and $x_{0}$ respectively, such that the evaluation map $e v_{\left(\sigma_{0}, x_{0}\right)}: U \rightarrow V$ is well defined. Under the hypotheses of Theorem 5. $\Sigma \cap(U \times V)$ is the vanishing locus of the map

$$
F:(\sigma, y) \in U \times V \mapsto\left(\sigma(y), \nabla^{L} \sigma_{\mid y}\right) \in L_{y}^{d} \times\left(K_{y}^{*} \otimes L_{y}^{d}\right) .
$$

It follows that for every $\sigma \in U, F\left(\sigma, e v_{\left(\sigma_{0}, x_{0}\right)}(\sigma)\right)=0$. By hypothesis the connection $\nabla^{X}$ restricts to a connection on the subbundle $K^{*}$. Hence, the connection $\nabla^{X, L}$ restricts to a connection on $K^{*} \otimes L^{d}$, denoted below by $D_{2}^{X, L}$. The latter makes it possible to differentiate $F$ with respect to the second variable. After differentiation we deduce that

$$
d_{1} F_{\mid\left(\sigma_{0}, x_{0}\right)}+D_{2}^{X, L} F_{\mid\left(\sigma_{0}, x_{0}\right)} \circ d_{\mid \sigma_{0}} e v_{\left(\sigma_{0}, x_{0}\right)}=0
$$

where $d_{1} F$ and $D_{2}^{X, L}$ denote the partial derivatives of $F$ with respect to the first and second variables respectively. Hence the relation

$$
d_{\mid \sigma_{0}} e v_{\left(\sigma_{0}, x_{0}\right)}=-\left(D_{2}^{X, L} F\right)_{\mid\left(\sigma_{0}, x_{0}\right)}^{-1} \circ d_{1} F_{\mid\left(\sigma_{0}, x_{0}\right)} .
$$

But the matrix of $D_{2}^{X, L} F \in \operatorname{End}\left(K_{x_{0}}^{\perp} \oplus K_{x_{0}}, L_{x_{0}}^{d} \oplus\left(K_{x_{0}}^{*} \otimes L_{x_{0}}^{d}\right)\right)$ at the point $\left(\sigma_{0}, x_{0}\right)$ is trigonal of the form $\left(\begin{array}{cc}\nabla^{L} \sigma_{0} & 0 \\ * & \nabla^{2} \sigma_{0}\end{array}\right)$, so that

$$
\operatorname{det} D_{2}^{X, L} F_{\mid\left(\sigma_{0}, x_{0}\right)}=\nabla^{L} \sigma_{0} \wedge \operatorname{det}\left(\nabla^{2} \sigma_{0}\right) .
$$

Likewise, let $\dot{\sigma}_{0}$ be a Bergman section at $x_{0}$, that is a unitary vector in the orthogonal complement of $H_{x_{0}}$ in $H^{0}\left(X, L^{d}\right)$. The restriction of

$$
d_{1} F \in \operatorname{End}\left(<\dot{\sigma}_{0}>\oplus H_{x_{0}} / \pi_{1}\left(\pi_{2}^{-1}\left(x_{0}\right)\right), L_{x_{0}}^{d} \oplus\left(K_{x_{0}}^{*} \otimes L_{x_{0}}^{d}\right)\right)
$$

at the point $\left(\sigma_{0}, x_{0}\right)$ to the orthogonal complement of $\pi_{1}\left(\pi_{2}^{-1}\left(x_{0}\right)\right)$ in $H^{0}\left(X, L^{d}\right)$ has the matrix $\left(\begin{array}{cc}\dot{\sigma}_{0}\left(x_{0}\right) & 0 \\ * & \nabla^{L}\end{array}\right)$, so that

$$
\operatorname{det} d_{1} F_{\mid\left(\sigma_{0}, x_{0}\right)}=\dot{\sigma}_{0}\left(x_{0}\right) \operatorname{det}\left(\nabla^{L}\right) .
$$

Taking the quotient, we deduce the result under the hypotheses of Theorem 5. The proof goes along the same lines under the hypotheses of Theorem 6 . 


\subsection{Proofs of Theorems 5 and 6}

Lemma 5 Under the hypotheses of Theorem [6, let $(\sigma, x) \in \Sigma_{i}$. Let $\phi_{x}: \mathbb{R} L_{x} \rightarrow \mathbb{R}$ be an isomorphism such that $\phi_{x} \circ \nabla_{\mid x}^{L} \sigma=-d p_{x}$. Then, $\phi_{x} \circ \nabla^{2} \sigma_{\mid K_{x}}=\nabla^{2}\left(p_{\mid \mathbb{R} C_{\sigma}}\right)_{\mid x}$, so that the quadratic form $\phi_{x} \circ \nabla^{2} \sigma_{\mid K_{x}}$ is non-degenerated of index $i$.

Proof. Let $v, w$ be vector fields on $\mathbb{R} C_{\sigma}$ at the neighbourhood of $x$. By definition, $0=\nabla_{v}^{L}\left(\nabla_{w}^{L} \sigma\right)=\nabla_{v, w}^{2} \sigma+\nabla_{\nabla_{v}^{X} w}^{L} \sigma$, so that

$$
\phi_{x} \circ \nabla_{v, w}^{2} \sigma=-\phi_{x} \circ \nabla_{\nabla_{v}^{X} w}^{L} \sigma=d p_{\mid x}\left(\nabla_{v}^{X} w\right) .
$$

Applying the same equality to the function $p$, we get

$$
d_{v}\left(d_{w} p\right)=\nabla^{X}(d p)(v, w)+d p\left(\nabla_{v}^{X} w\right)=d p\left(\nabla_{v}^{X} w\right)
$$

by hypothesis on $\nabla^{X}$, so that $\phi_{x} \circ \nabla_{v, w}^{2} \sigma=d_{v}\left(d_{w} p\right)$. Finally, applying this equality to the restriction $p_{\mid \mathbb{R} C_{\sigma}}$, we get

$$
d_{v}\left(d_{w} p\right)_{\mid x}=\nabla_{v, w}^{2} p_{\left|\mathbb{R} C_{\sigma}\right| x}+d p_{\mid \mathbb{R} C_{\sigma}}\left(\nabla_{v}^{C_{\sigma}} w\right)_{\mid x}=\nabla_{v, w}^{2}\left(p_{\mid \mathbb{R} C_{\sigma}}\right)_{\mid x}
$$

where $\nabla^{C_{\sigma}}$ denotes any connexion on $T \mathbb{R} C_{\sigma}$. Hence the result.

Proposition 3 Under the hypotheses of Theorem 5 ,

$$
\frac{1}{d^{n}} \int_{\pi_{1}\left(\pi_{2}^{-1}(x)\right)} \lambda_{(\sigma, x)} \wedge \overline{\lambda_{(\sigma, x)}} d \mu_{\mathbb{C}}(\sigma)=\left(\pi^{n} e_{\mathbb{C}}(n-1)+O\left(\frac{1}{\sqrt{d}}\right)\right) d v o l_{h}
$$

whatever the normalized volume form $d x$ chosen on of $X$ to define $d \mu_{\mathbb{C}}$ is (see $\oiint_{1.1}$ ), and where $O\left(\frac{1}{\sqrt{d}}\right) \in L^{1}\left(X\right.$, dvol $\left._{h}\right)$ denotes a sequence of integrable functions having pole at $\operatorname{Crit}(p)$ (resp. Base $(p))$ of order at most $2 n-2$ (resp. 2). Likewise, under the hypotheses of Theorem 6 ,

$$
\frac{1}{\sqrt{d}^{n}} \int_{\pi_{1}\left(\pi_{2}^{-1}(x)\right)}\left|\lambda_{(\sigma, x)}\right| d \mu_{\mathbb{R}}(\sigma)=\left(\sqrt{\pi}^{n-1} e_{\mathbb{R}}(i, n-1-i)+O\left(\frac{1}{\sqrt{d}}\right)\right) d v o l_{h}
$$

whatever the normalized volume form chosen on $X$ is, and where $O\left(\frac{1}{\sqrt{d}}\right) \in L^{1}\left(\mathbb{R} X, d\right.$ vol $\left._{h}\right)$ denotes a sequence of integrable functions having poles at Crit $(p)$ of order at most $n-1$.

In Proposition 3, a function $f \in L^{1}\left(X, d v o l_{h}\right)$ is said to have a pole of order at most $k$ along a submanifold $Y$ if $r^{k} f$ is bounded near $Y$, where $r$ denotes the distance function to $Y$.

Proof. Let $x \in X \backslash(\operatorname{Crit}(p) \cup \operatorname{Base}(p))$ (resp. $x \in \mathbb{R} X \backslash \operatorname{Crit}(p))$ and $\left(x_{1}, \cdots, x_{n}\right)$ be local holomorphic (resp. real holomorphic) coordinates in the neighbourhood of $x=(0, \cdots, 0)$ such that $\left(\frac{\partial}{\partial x_{1}}, \cdots, \frac{\partial}{\partial x_{n}}\right)$ be orthonormal at $x$ and $\left(\frac{\partial}{\partial x_{2}}, \cdots, \frac{\partial}{\partial x_{n}}\right)$ spans $\operatorname{ker}(d p)$ at the point $x$. With the notations of $\$ 1.3$, every element $\sigma \in H^{0}\left(X, L^{d}\right)$ (resp. $\sigma \in \mathbb{R} H^{0}\left(X, L^{d}\right)$ ) writes

$$
\sigma=\sum_{j=0}^{n} a_{j} \sigma_{j}+\sum_{1 \leq k \leq l \leq n} b_{k l} \sigma_{k l}+\tau,
$$


where $a_{j}, b_{k l} \in \mathbb{C}\left(\right.$ resp. $\left.a_{j}, b_{k l} \in \mathbb{R}\right)$ and $\tau \in H_{3 x}$ (resp. $\tau \in \mathbb{R} H_{3 x}$ ), see (2) (resp. (3) ). In the previous equality, $\sigma \in \pi_{1}\left(\pi_{2}^{-1}(x)\right)$ if and only if $a_{j}=0$ for every $j \in$ $\{0, \cdots, n\} \backslash\{1\}$ and we assume that this holds true. Moreover, from Lemmas 1 and 3 ,

$$
\begin{aligned}
\sigma_{0} & =\lambda_{0} e^{d}(x)\left(1+O\left(\frac{1}{d^{6}}\right)\right), \\
\forall j \in\{1, \cdots, n\}, \nabla^{L} \sigma_{j \mid x} & =\sqrt{\pi d} \lambda_{0} e^{d}(x)\left(1+O\left(\frac{1}{d^{6}}\right)\right) d x_{j} \text { and } \\
\nabla^{2} \sigma_{j j \mid x} & =\frac{\pi d \lambda_{0}}{\sqrt{2}} e^{d}(x)\left(1+O\left(\frac{1}{d^{6}}\right)\right)\left(2 d x_{j} \otimes d x_{j}\right), \text { while } \\
\forall 1 \leq k<l \leq n, \nabla^{L} \sigma_{k l \mid x} & =0 \text { and } \\
\nabla^{2} \sigma_{k l \mid x} & =\pi d \lambda_{0} e^{d}(x)\left(1+O\left(\frac{1}{d^{6}}\right)\right)\left(d x_{k} \otimes d x_{l}+d x_{l} \otimes d x_{k}\right) .
\end{aligned}
$$

These equations do not depend on the chosen connexions $\nabla^{L}, \nabla^{X}$. It follows that

$$
\nabla^{2} \sigma_{1 \mid K_{x}}=\sqrt{\pi d} \lambda_{0} e^{d}(x)\left(1+O\left(\frac{1}{d^{6}}\right)\right) \nabla^{X}\left(d x_{1}\right)
$$

since by hypothesis, the restriction of $d x_{1}$ to $K_{x}$ vanishes. Likewise,

$$
\frac{1}{\pi d \lambda_{0}} \frac{\nabla^{2} \sigma_{\mid K_{x}}}{e^{d}(x)}=\sum_{j=2}^{n} \frac{b_{j j}}{\pi d \lambda_{0}} \frac{\nabla^{2} \sigma_{j j \mid K_{x}}}{e^{d}(x)}+\sum_{2 \leq k<l \leq n} \frac{b_{k l}}{\pi d \lambda_{0}} \frac{\nabla^{2} \sigma_{k l \mid K_{x}}}{e^{d}(x)}+\frac{a_{1}}{\pi d \lambda_{0}} \frac{\nabla^{2} \sigma_{1 \mid K_{x}}}{e^{d}(x)},
$$

so that this restriction writes

$$
\sum_{j=2}^{n} \sqrt{2} b_{j j} d x_{j} \otimes d x_{j}+\sum_{2 \leq k<l \leq n} b_{k l}\left(d x_{k} \otimes d x_{l}+d x_{l} \otimes d x_{k}\right)+O\left(\frac{1}{\sqrt{d}}\right) .
$$

Using the notations of $\$ 1.1$, let $B$ be the matrix $\sum_{2 \leq k \leq l \leq n} b_{k l} \widetilde{E}_{k l}$, and let us first assume that $d x=\frac{1}{\int_{X} \omega^{n}} \omega^{n}$, so that from Lemma 4], the sections $\left(\sigma_{j}\right)_{0 \leq j \leq n}$ and $\left(\sigma_{k l}\right)_{0 \leq k \leq l \leq n}$ are asymptotically orthonormal. We deduce that pointwise on $X \backslash$ $(\operatorname{Crit}(p) \cup \operatorname{Base}(p))$,

$$
\frac{1}{\sqrt{\pi d}^{n-1}}\left|\frac{\operatorname{det}\left(\nabla^{2} \sigma_{\mid K_{x}}\right)}{\operatorname{det}\left(\nabla_{\frac{\partial}{\partial x_{k}}}^{L} \sigma_{l \mid x}\right)_{2 \leq k \leq l \leq n}}\right|=|\operatorname{det} B|\left|d x_{2} \wedge \cdots \wedge d x_{n}\right|+O\left(\frac{1}{\sqrt{d}}\right) .
$$

Moreover,

$$
\frac{1}{\sqrt{\pi d}} \frac{\nabla^{L} \sigma}{\sigma_{0}(x)}=a_{1} d x_{1}+O\left(\frac{1}{d^{6}}\right)
$$

so that

$$
\frac{1}{(\pi d)^{n}} \lambda_{(\sigma, x)} \wedge \overline{\lambda_{(\sigma, x)}}=\left(\left|a_{1}\right|^{2}|\operatorname{det} B|^{2}+O\left(\frac{1}{\sqrt{d}}\right)\right) d v o l_{h \mid x} .
$$

Now, we decompose the space $\pi_{1}\left(\pi_{2}^{-1}(x)\right)$ as

$$
\pi_{1}\left(\pi_{2}^{-1}(x)\right)=\left(H_{3 x} \cap \pi_{1}\left(\pi_{2}^{-1}(x)\right)\right) \oplus\left(H_{3 x} \cap \pi_{1}\left(\pi_{2}^{-1}(x)\right)\right)^{\perp}
$$

(resp. $\left.\pi_{1}\left(\pi_{2}^{-1}(x)\right)=\left(\mathbb{R} H_{3 x} \cap \pi_{1}\left(\pi_{2}^{-1}(x)\right)\right) \oplus\left(\mathbb{R} H_{3 x} \cap \pi_{1}\left(\pi_{2}^{-1}(x)\right)\right)^{\perp}\right)$, see (21) and (31). 
Denote by $H^{\prime}$ the vector space spanned by $\sigma_{1}$ and $\sigma_{k, l}, 1 \leq k \leq l \leq n$ and by

$$
\pi^{\prime}:\left(H_{3 x} \cap \pi_{1}\left(\pi_{2}^{-1}(x)\right)\right)^{\perp} \rightarrow H^{\prime}
$$

the projection onto $H^{\prime}$ directed by $H_{3 x}\left(\right.$ resp. $\left.\mathbb{R} H_{3 x}\right)$. We deduce that

$$
\begin{aligned}
\frac{1}{d^{n}} \int_{\pi_{1}\left(\pi_{2}^{-1}(x)\right)} \lambda_{(\sigma, x)} \wedge \overline{\lambda_{(\sigma, x)}} d \mu_{\mathbb{C}}(\sigma)= & \frac{1}{d^{n}} \int_{\left(H_{3 x} \cap \pi_{1}\left(\pi_{2}^{-1}(x)\right)\right)^{\perp}} \lambda_{(\sigma, x)} \wedge \overline{\lambda_{(\sigma, x)}} d \mu_{\mathbb{C}}(\sigma) \\
= & \pi^{n} \int_{H^{\prime}}\left|a_{1}\right|^{2}|\operatorname{det} B|^{2}\left(\pi_{*}^{\prime} d \mu_{\mathbb{C}}\right)\left(a_{1}, B\right) \\
& +O\left(\frac{1}{\sqrt{d}}\right) d v o l_{h \mid x}
\end{aligned}
$$

From Lemma 4, the pushforward measure $\pi_{*}^{\prime} \mu_{\mathbb{C}}$ coincides with the Gaussian measure on the space with coordinates $a_{1}$ and $\left(b_{k l}\right)_{1 \leq k \leq l \leq n}$ up to a $O\left(\frac{1}{\sqrt{d}}\right)$ term. Hence,

$$
\begin{aligned}
\frac{1}{d^{n}} \int_{\pi_{1}\left(\pi_{2}^{-1}(x)\right)} \lambda_{(\sigma, x)} \wedge \overline{\lambda_{(\sigma, x)}} d \mu_{\mathbb{C}}(\sigma) & =\pi^{n}\left(\int_{S y m(n-1, \mathbb{C})}|\operatorname{det} B|^{2} d \mu_{\mathbb{C}}(B)+O\left(\frac{1}{\sqrt{d}}\right)\right) d v o l_{h \mid x} \\
& =\left(\pi^{n} e_{\mathbb{C}}(n-1)+O\left(\frac{1}{\sqrt{d}}\right)\right) d v o l_{h \mid x} .
\end{aligned}
$$

This result remains unchanged if a different normalized volume form $d x$ is used on $X$ to define the $L^{2}$-scalar product, since from Remark 3 , this asymptotically just has the effect of dilating the scalar product on the subspace $\left(H_{3 x} \cap \pi_{1}\left(\pi_{2}^{-1}(x)\right)\right)^{\perp}$, while from Remark 4 and Proposition 2, such a dilation does not affect the integral $\int_{\left(H_{3 x} \cap \pi_{1}\left(\pi_{2}^{-1}(x)\right)\right)^{\perp}} \lambda_{(\sigma, x)} \wedge \overline{\lambda_{\sigma, x}} d \mu_{\mathbb{C}}(\sigma)$.

Since $\nabla^{X}$ is not defined at the critical and base points of $p$, we have now to estimate the singularities of $\nabla^{X}\left(d x_{1}\right)$ near these loci. In the coordinates $\left(x_{1}, \cdots, x_{n}\right)$ around $x$, let us write $d p=\sum_{i=1}^{n} \alpha_{i} d x_{i}$, so that at the point $x, \alpha_{2}(x)=\cdots=\alpha_{n}(x)=0$ and $\left|\alpha_{1}(x)\right|=|| d p_{x} \|$. Then,

$$
0=\nabla^{X}(d p)_{\mid K_{x}}=\alpha_{1}\left(\nabla^{X} d x_{1}\right)_{\mid K_{x}}+\sum_{i=1}^{n}\left(d \alpha_{i} \otimes d x_{i}\right)_{\mid K_{x}}
$$

so that $\left\|\nabla^{X} d x_{1 \mid K_{x}}\right\|=\frac{1}{\| d p_{|x|} \mid}\left\|\sum_{i=1}^{n} d \alpha_{i} \otimes d x_{i \mid K_{x}}\right\|$ has a pole of order one at $x$, since by definition of a Lefschetz pencil, $d p$ vanishes transversally at $x$. By developing the determinant of $\nabla^{2} \sigma$ and using (5), we thus get that

$$
\frac{1}{(\pi d)^{n}} \lambda_{(\sigma, x)} \wedge \overline{\lambda_{(\sigma, x)}}=\left(\left|a_{1}\right|^{2}|\operatorname{det} B|^{2}+O\left(\frac{1}{\sqrt{d}}\right)\right) d v o l_{h}
$$

where for every $d>0$, the singularities of the function $O\left(\frac{1}{\sqrt{d}}\right)$, which is polynomial in $a_{1}$ and $b_{k, l}$ for $2 \leq k \leq l \leq n$, are poles of order at most $2(n-1)$ near the critical points.

Near the base points, these are poles of order at most 2. Indeed, the normal form for $p$ near a base point writes $p:\left(y_{1}, \cdots, y_{n}\right) \in \mathbb{C}^{n} \mapsto y_{1} / y_{2} \in \mathbb{C}$, so that

$$
d p_{\mid\left(y_{1}, \cdots, y_{n}\right)}=\frac{y_{2} d y_{1}-y_{1} d y_{2}}{y^{2}}
$$


is not well defined along $y_{2}=0$. Denote by $\beta$ the numerator one-form $y_{2} d y_{1}-y_{1} d y_{2}$, which is well defined everywhere. When the point $x$ lies in such a chart, there is no obstruction in finding local coordinates around $x$ which are orthonormal at $x$ and such that in these coordinates, $\beta$ writes $\alpha_{1} d x_{1}+\alpha_{2} d x_{2}$ where $\alpha_{1}, \alpha_{2}$ only depend on $x_{1}, x_{2}, \alpha_{2}(x)=0$ and $\alpha_{1}(x)$ is a function of $x$ having a simple zero along the base locus. Then, by hypothesis on $\nabla^{X}$,

$$
0=\nabla^{X} \beta_{\mid K_{x}}=\alpha_{1} \nabla^{X}\left(d x_{1}\right)_{\mid K_{x}}+\frac{\partial \alpha_{2}}{\partial x_{2}} d x_{2} \otimes d x_{2 \mid K_{x}} .
$$

It follows that $\nabla^{X}\left(d x_{1}\right)_{\mid K_{x}}$ has a simple pole along the base locus, and by (44) the matrix of $\nabla^{2} \sigma_{1 \mid K_{x}}$ in the basis $\left(\frac{\partial}{\partial x_{2}}, \cdots, \frac{\partial}{\partial x_{n}}\right)$ is elementary, with only one diagonal coefficient having a simple pole along the base locus. After developing the determinant, we deduce that the $O\left(\frac{1}{\sqrt{d}}\right)$ function has a pole of order at most 2 near the base locus.

In the real case, finally we get likewise that

$$
\frac{1}{\sqrt{\pi d}^{n}}\left|\lambda_{(\sigma, x)}\right|=\left(\left|a_{1}\right||\operatorname{det} B|+O\left(\frac{1}{\sqrt{d}}\right)\right) d v o l_{h \mid x}
$$

where the singularities of the functions $O\left(\frac{1}{\sqrt{d}}\right)$ are poles of orders at most $n-1$ near the critical points. After integration, we deduce that

$\frac{1}{\sqrt{d}^{n}} \int_{\pi_{1}\left(\pi_{2}^{-1}(x)\right)}\left|\lambda_{(\sigma, x)}\right| d \mu_{\mathbb{R}}(\sigma)=\sqrt{\pi}^{n}\left(\int_{H_{i}^{\prime}}\left|a_{1}\right||\operatorname{det} B|\left(\pi_{*}^{\prime} d \mu_{\mathbb{R}}\left(a_{1}, B\right)\right)+O\left(\frac{1}{\sqrt{d}}\right)\right) d v o l_{h \mid x}$,

where from Lemma [5, $H_{i}^{\prime}=\left\{\sigma \in H^{\prime} \mid \phi_{x} \circ \nabla^{2} \sigma_{\mid K_{x}}\right.$ is of index $\left.i\right\}$. Again, we deduce from Lemma 4 that

$$
\begin{aligned}
\frac{1}{\sqrt{d}^{n}} \int_{\pi_{1}\left(\pi_{2}^{-1}(x)\right)}\left|\lambda_{(\sigma, x)}\right| d \mu_{\mathbb{R}}(\sigma)= & \sqrt{\pi}^{n} \int_{\mathbb{R}}\left|a_{1}\right| d \mu_{\mathbb{R}}\left(a_{1}\right) \\
& \left(\int_{\text {Sym }(i, n-1-i, \mathbb{R})}|\operatorname{det} B| d \mu_{\mathbb{R}}(B)+O\left(\frac{1}{\sqrt{d}}\right)\right)\left|d v o l_{h \mid x}\right| \\
= & \left(\sqrt{\pi}^{n-1} e_{\mathbb{R}}(i, n-1-i)+O\left(\frac{1}{\sqrt{d}}\right)\right)\left|d \operatorname{dvol}_{h \mid x}\right|,
\end{aligned}
$$

and this result remains unchanged if a different normalized volume form $d x$ is used on $X$.

Proof of Theorems 5 and 6. From Proposition 3 follows that under the hypotheses of Theorem 5 (resp. of Theorem 6 ), the measure

$$
\frac{1}{d^{n}} \int_{\pi_{1}\left(\pi_{2}^{-1}(x)\right)} \lambda_{(\sigma, x)} \wedge \overline{\lambda_{(\sigma, x)}} d \mu_{\mathbb{C}}(\sigma)\left(\text { resp. } \frac{1}{\sqrt{d}^{n}}\left|\lambda_{(\sigma, x)}\right| d \mu_{\mathbb{R}}(\sigma)\right)
$$

weakly converges to the measure

$$
\pi^{n} e_{\mathbb{C}}(n-1) \operatorname{dvol}_{h}\left(\operatorname{resp} . \sqrt{\pi}^{n-1} e_{\mathbb{R}}(i, n-1-i) d v o l_{h}\right) .
$$

Theorems 5 and 6 then follow from Propositions 1 and 2 , whatever the normalized volume form $d x$ is chosen on $X$ to define the $L^{2}$-scalar product $\langle.,$.$\rangle .$ 


\section{Expected determinant of random symmetric matrices}

In 2.1 we study the asymptotic distribution of $e_{\mathbb{R}}(p, q)$ for large $n=p+q$. We then compute $e_{\mathbb{C}}(n)$ in $\$ 2.2 .1$ and $e_{\mathbb{R}}(n)$ in $\S \$ 2.2 .2$ and 2.2.3. We also give in $\$ 2.2 .4$ the values of $e_{\mathbb{R}}(p, q)$ for $p+q \leq 3$.

\subsection{Large random real symmetric matrices}

\subsubsection{The energy functional}

Let

$$
\begin{aligned}
& f: \mathbb{R}^{2} \longrightarrow \mathbb{R} \cup\{\infty\} \\
& (x, y) \longmapsto \begin{cases}\frac{1}{2}\left(x^{2}+y^{2}\right)-\log |x-y| & \text { if } x \neq y \\
+\infty & \text { if } x=y\end{cases}
\end{aligned}
$$

Let $\mathcal{M}_{1}^{+}(\mathbb{R})$ be the space of probability measures on $\mathbb{R}$ and $H$ be the energy functional defined by the relation:

$$
\begin{aligned}
H: \mathcal{M}_{1}^{+}(\mathbb{R}) & \longrightarrow \mathbb{R} \cup\{\infty\} \\
\mu & \longmapsto \begin{cases}\frac{1}{2} \iint_{\mathbb{R}^{2}} f(x, y) d \mu(x) d \mu(y) & \text { if } \int_{\mathbb{R}} \log (|x|+1) d \mu(x)<+\infty \\
+\infty & \text { otherwise. }\end{cases}
\end{aligned}
$$

This functional is lower semicontinuous, strictly convex and reaches its unique minimum at the semi-circle law $\mu_{W}$ of Wigner, see $\S 2.6 .1$ of [1]. Moreover, $H\left(\mu_{W}\right)=$ $\frac{1}{4}\left(\frac{3}{2}+\log 2\right)$.

For every $0 \leq \alpha \leq 1$, define

$$
\mathcal{M}_{\alpha, 1-\alpha}^{+}(\mathbb{R})=\left\{\mu \in \mathcal{M}_{1}^{+}(\mathbb{R}) \mid \mu\left(\mathbb{R}_{-}^{*}\right)=\alpha \text { and } \mu\left(\mathbb{R}_{+}^{*}\right)=1-\alpha\right\} .
$$

Since the functional $H$ is strictly convex and equals $+\infty$ on atomic measures, its restriction to $\mathcal{M}_{\alpha, 1-\alpha}^{+}$reaches its minimum at a unique measure $\mu_{\alpha} \in \mathcal{M}_{\alpha, 1-\alpha}^{+}$which has no atom. In particular, $\mu_{\frac{1}{2}}=\mu_{W}$. For every $0 \leq \alpha \leq 1$, we set

$$
m_{\alpha}=\min _{\mathcal{M}_{\alpha, 1-\alpha}^{+}(\mathbb{R})} H=H\left(\mu_{\alpha}\right) .
$$

Lemma 6 The function $m: \alpha \in[0,1] \rightarrow m_{\alpha} \in \mathbb{R}_{+}$is strictly decreasing over $[0,1 / 2]$ and strictly increasing over $[1 / 2,1]$. More precisely, for every $\alpha \in[0,1] \backslash\{1 / 2\}$, there exists $c_{\alpha}>0$ such that $\forall t \in[0,1], m_{t \alpha+(1-t) \frac{1}{2}} \leq m_{\alpha}+\left(t^{2}-1\right) c_{\alpha}$.

Proof. Let $\alpha \in[0,1] \backslash\left\{\frac{1}{2}\right\}$ and $f^{\alpha} \in L^{1}(\mathbb{R}, d x)$ be the density of $\mu_{\alpha}$ with respect to the Lebesgue measure $d x$. We decompose $f^{\alpha}=f_{p}+f_{i}$ into odd and even functions, so that $f_{p}=\frac{1}{2}\left(f^{\alpha}+f^{\alpha} \circ(-I d)\right)$ and $f_{i}=\frac{1}{2}\left(f^{\alpha}-f^{\alpha} \circ(-I d)\right)$. Likewise, we set $\mu_{p}=f_{p} d x$ and $\mu_{i}=f_{i} d x$, so that $\mu^{\alpha}=\mu_{p}+\mu_{i}$. Then, for every $t \in[-1,1]$,

$$
\begin{aligned}
H\left(\mu_{p}+t \mu_{i}\right)= & \frac{1}{2} \iint_{\mathbb{R}^{2}} f(x, y)\left(d \mu_{p}(x) d \mu_{p}(y)+t d \mu_{p}(x) d \mu_{i}(y)+t d \mu_{i}(x) d \mu_{p}(y)\right) \\
& +\frac{t^{2}}{2} \iint_{\mathbb{R}^{2}} f(x, y) d \mu_{i}(x) d \mu_{i}(y)=H\left(\mu_{p}\right)+t^{2} H\left(\mu_{i}\right)
\end{aligned}
$$


from Fubini's theorem, since $\int_{\mathbb{R}} \frac{1}{2}\left(x^{2}+y^{2}\right) d \mu_{i}(x)=\int_{\mathbb{R}} \frac{1}{2}\left(x^{2}+y^{2}\right) d \mu_{i}(y)=0$ while likewise $\int_{\mathbb{R}} \log |y-x| d \mu_{p}(x)$ and $\int_{\mathbb{R}} \log |y-x| d \mu_{p}(y)$ are even functions of $y$ and $x$ respectively. Since $H$ is strictly convex, so does its restriction over the interval $\left\{\mu_{p}+t \mu_{i}, t \in[-1,1]\right\}$, so that $H\left(\mu_{i}\right)$ has to be positive. However, for every $t \in[0,1]$, $\mu_{p}+t \mu_{i}$ belongs to $\mathcal{M}_{\left(t \alpha+(1-t) \frac{1}{2},(1+t) \frac{1}{2}-t \alpha\right)}^{+}(\mathbb{R})$ and we deduce that $m_{t \alpha+(1-t) \frac{1}{2}} \leq H\left(\mu_{p}\right)+$ $t^{2} H\left(\mu_{i}\right)=m_{\alpha}+\left(t^{2}-1\right) H\left(\mu_{i}\right)$. Hence the result.

Remark 5 It would be of interest to compute explicitely the function $\alpha \in[0,1] \mapsto$ $m_{\alpha} \in\left[m_{\frac{1}{2}}, \infty\right]$ and in particular its asymptotics near $\alpha=1 / 2$. Likewise, we saw in the proof of Lemma [6] that the functional $H$ restricted to measures of type $g(x) d x$, where $g$ is an odd function in $L^{1}(\mathbb{R}, d x)$ with $\int_{\mathbb{R}^{+}} g d x=1$, is positive, but does its infinimum remain positive? Finally, what is the measure $\mu_{\alpha}$ ? (see [5] for the case $\alpha=0)$

\subsubsection{Measure concentration around matrices of vanishing signature}

Proposition 4 For every $\alpha \in\left[0,1 / 2\left[\right.\right.$, there exists $c_{\alpha}>0$ such that

$$
\sum_{i=0}^{\lfloor\alpha n\rfloor} e_{\mathbb{R}}(i, n-i) \leq \exp \left(-c_{\alpha} n^{2}\right)
$$

and $\mu_{\mathbb{R}}\left(\bigcup_{i=0}^{\lfloor\alpha n\rfloor} \operatorname{Sym}(i, n-i, \mathbb{R})\right) \leq \exp \left(-c_{\alpha} n^{2}\right)$.

Proof. The orthogonal group $O_{n}(\mathbb{R})$ acts by conjugation on real symmetric matrices and a fundamental domain for this action is given by diagonal matrices with stabilizer $\{ \pm 1\}^{n}$. From the coarea formula (see Theorem 3.2.3 of [10] or Theorem 1 of [21]), we deduce that for every $0 \leq i \leq n$,

$$
e_{\mathbb{R}}(i, n-i)=\frac{V o l\left(O_{n}(\mathbb{R})\right)}{2^{n} \sqrt{\pi}^{\frac{n(n-1)}{2}}} \int_{\substack{\lambda_{1}<\cdots<\lambda_{i}<0 \\ 0<\lambda_{i+1}<\cdots<\lambda_{n}}}\left|\prod_{i=1}^{n} \sqrt{2} \lambda_{i}\right| \prod_{1 \leq i<j \leq n}\left|\lambda_{j}-\lambda_{i}\right| d \mu(\lambda),
$$

where the volume of $O_{n}(\mathbb{R})$ is computed with respect to the right invariant metric for which the basis $\left(E_{i j}-E_{j i}\right)_{1 \leq i<j \leq n}$ of its Lie algebra is orthonormal, see $\$ 2.1 .3$, and where $d \mu(\lambda)$ denotes the Gaussian measure on $\mathbb{R}^{n}$. As a consequence, for every $0 \leq i \leq n$,

$$
\begin{aligned}
e_{\mathbb{R}}(i, n-i)= & \frac{\operatorname{Vol}\left(O_{n}(\mathbb{R})\right)}{\sqrt{2}^{n} \sqrt{\pi}^{\frac{n(n+1)}{2}}} \\
& \int_{\substack{\lambda_{1}<\cdots<\lambda_{i}<0 \\
0<\lambda_{i+1}<\cdots<\lambda_{n}}} \exp \left(-\sum_{j=1}^{n} \lambda_{j}^{2}+\sum_{\substack{1 \leq j<k \leq n \\
=}} \operatorname{cog} \mid \lambda_{j} n ! \int_{\substack{\gamma_{1}<\cdots<\gamma_{i}<0 \\
0<\gamma_{i+1}<\cdots<\gamma_{n}}} \exp \left(\sum_{1 \leq j<k \leq n} \log \left|\gamma_{j}-\gamma_{k}\right|-\frac{1}{2} \sum_{1 \leq j<k \leq n}^{n}\left(\left|\lambda_{j}\right| d \lambda_{j}\right)\right.\right. \\
& \exp \left(-\frac{1}{2} \sum_{j=1}^{n} \gamma_{j}^{2}\right) \prod_{j=1}^{n}\left(\left|\gamma_{j}\right| d \gamma_{j}\right)
\end{aligned}
$$


where

$$
c_{n}=\frac{\operatorname{Vol}\left(O_{n}(\mathbb{R})\right)}{n ! \sqrt{2}^{n} \sqrt{\pi}^{\frac{n(n+1)}{2}}}\left(\frac{n}{2}\right)^{\frac{n(n-1)}{4}+n},
$$

and where we wrote, for every $1 \leq j \leq n, \lambda_{j}=\sqrt{\frac{n}{2}} \gamma_{j}$. We now proceed as in $\S 3.1$ of [3] (or $\S 2.6 .1$ of [1]). Define, for every $\gamma_{1}<\cdots<\gamma_{i}<0<\gamma_{i+1}<\cdots<\gamma_{n}$,

$$
\mu_{n}=\frac{1}{n} \sum_{j=1}^{n} \delta_{\gamma_{j}} \in \mathcal{M}_{i / n, 1-i / n}^{+}(\mathbb{R}),
$$

so that

$$
\sum_{1 \leq j<k \leq n}\left|\gamma_{j}-\gamma_{k}\right|-\frac{1}{2} \sum_{1 \leq j<k \leq n}\left(\gamma_{j}^{2}+\gamma_{k}^{2}\right)=-n^{2} \iint_{x<y} f(x, y) d \mu_{n}(x) d \mu_{n}(y) .
$$

Let $M>m_{0}, f^{M}=\min (f, M)$ and

$$
\forall \mu \in \mathcal{M}_{1}^{+}(\mathbb{R}), H_{M}(\mu)=\frac{1}{2} \iint_{\mathbb{R}^{2}} f^{M}(x, y) d \mu(x) d \mu(y) .
$$

By Lemma 6,

$$
\min _{\bigcup_{\beta \leq \alpha} \mathcal{M}_{\beta, 1-\beta}^{+}(\mathbb{R})} H_{M}=\min _{\bigcup_{\beta \leq \alpha} \mathcal{M}_{\beta, 1-\beta}^{+}(\mathbb{R})} H .
$$

As a consequence, for every $0 \leq i \leq\lfloor\alpha n\rfloor$,

$$
\iint_{x<y} f(x, y) d \mu_{n}(x) d \mu_{n}(y)=H_{M}\left(\mu_{N}\right)-\frac{M}{2 n} \geq m_{\alpha}-\frac{M}{2 n} .
$$

Moreover, by Lemma 8 (see $\$ 2.1 .3$ below) and Stirling's formula, $\ln \left(c_{n}\right)=n^{2} m_{1 / 2}+$ $O(n)$. Hence, there exists a constant $D>0$ such that

$$
\sum_{i=0}^{\lfloor\alpha n\rfloor} e_{\mathbb{R}}(i, n-i) \leq \exp \left(-n^{2}\left(m_{\alpha}-m_{1 / 2}\right)+D n\right)\left(\int_{\mathbb{R}}|\gamma| e^{-\frac{\gamma^{2}}{2}} d \gamma\right)^{n},
$$

and the first part of Proposition 4 follows. The proof of the second part goes along the same lines.

\subsubsection{Volume of the orthogonal group}

Let us equip the vector space of real antisymmetric matrices with the scalar product turning the basis $\left(E_{i j}-E_{j i}\right)_{1 \leq i<j \leq n}$ into an orthonormal one. This scalar product on the Lie algebra of $O_{n}(\mathbb{R})$ induces on $O_{n}(\mathbb{R})$ a Riemannian metric for which the multiplications on the right by elements produce isometries. We recall in the following Lemma 7 the value of the total volume of $O_{n}(\mathbb{R})$ for this metric.

Lemma 7 For every positive integer $n$,

$$
\operatorname{Vol}\left(O_{n}(\mathbb{R})\right)=\frac{n ! \sqrt{\pi}^{\frac{n(n+1)}{2}} \sqrt{2^{\frac{n(n-1)}{2}}}}{\prod_{j=1}^{n} \Gamma(1+j / 2)} .
$$


Proof. The coarea formula gives as in the proof of Proposition 4

$$
\operatorname{Vol}\left(O_{n}(\mathbb{R})\right)=\frac{2^{n} \sqrt{\pi}^{\frac{n(n-1)}{2}}}{\int_{\lambda_{1}<\cdots<\lambda_{n}} \prod_{1 \leq i<j \leq n}\left(\lambda_{j}-\lambda_{i}\right) d \mu(\lambda)} .
$$

But

$$
\int_{\lambda_{1}<\cdots<\lambda_{n}} \prod_{1 \leq i<j \leq n}\left(\lambda_{j}-\lambda_{i}\right) d \mu(\lambda)=\frac{1}{n ! \sqrt{2}^{\frac{n(n+1)}{2}} \sqrt{\pi}^{n}} \int_{\mathbb{R}^{n}}\left|\prod_{1 \leq i<j \leq n}\left(\lambda_{j}-\lambda_{i}\right)\right| e^{-\frac{\|\lambda\| \|^{2}}{2}} d \lambda .
$$

The latter integral can be computed using Selberg's formula, see Theorem 3.3.1 of [16], which writes

$$
\left.\int_{\mathbb{R}^{n}}\left|\prod_{1 \leq i<j \leq n}\left(\lambda_{j}-\lambda_{i}\right)\right| e^{-\frac{\|\lambda\|^{2}}{2}} d \lambda=2^{n} \sqrt{2}^{n} \prod_{j=1}^{n} \Gamma(1+j / 2)\right) .
$$

Hence the result.

Lemma 8 The following asymptotic development holds :

$$
\ln \left(\frac{\operatorname{Vol}\left(O_{n}(\mathbb{R})\right)}{\sqrt{2}^{n} \sqrt{\pi}^{\frac{n(n-1)}{2}}}\right)=-\frac{n^{2} \ln n}{4}+n^{2}\left(\frac{3}{8}+\frac{\ln 2}{2}\right)+\frac{1}{4} n \ln n+O(n) .
$$

Proof. From Lemma 7, when $n=2 m$ is even,

$$
\frac{\operatorname{Vol}\left(O_{n}(\mathbb{R})\right)}{\sqrt{2}^{n} \sqrt{\pi}^{\frac{n(n-1)}{2}}}=\frac{n ! \sqrt{\pi}^{n} \sqrt{2}^{\frac{n(n-3)}{2}}}{\prod_{j=1}^{n / 2}(j ! \Gamma(j+1 / 2))} .
$$

From Stirling's formula, $n$ ! is equivalent to $n^{n} e^{-n} \sqrt{2 \pi n}$ as $n$ grows to infinity and $\Gamma(j+1 / 2)$ to $(j-1) ! \sqrt{j-1}$. It follows that

$$
\begin{aligned}
\ln \left(\prod_{j=1}^{m}(j ! \Gamma(j+1 / 2))\right)= & 2 \sum_{j=1}^{m-1}\left(j \ln j-j+\frac{3}{4} \ln j\right)+m \ln m+O(n) \\
= & \sum_{j=1}^{m-1}\left((j+1)^{2} \ln (j+1)-j^{2} \ln j-3 j+\frac{1}{2}(j+1) \ln (j+1)-\frac{j}{2} \ln j\right) \\
& +m \ln m+O(n) \\
= & \frac{n^{2}}{4} \ln \left(\frac{n}{2}\right)-\frac{3}{8} n^{2}+\frac{3 n}{4} \ln \left(\frac{n}{2}\right)+O(n) .
\end{aligned}
$$

Finally, we obtain

$$
\ln \left(\frac{\operatorname{Vol}\left(O_{n}(\mathbb{R})\right)}{\sqrt{2}^{n} \sqrt{\pi}^{\frac{n(n-1)}{2}}}\right)=n \ln n+\frac{n^{2}}{4} \ln 2+\frac{n^{2}}{4} \ln 2-\frac{n^{2}}{4} \ln (n)+\frac{3}{8} n^{2}-\frac{3 n}{4} \ln (n)+O(n)
$$

and the result when $n$ is even. When $n=2 m+1$ is odd, we have

$$
\frac{V o l\left(O_{n}(\mathbb{R})\right)}{\sqrt{2}^{n} \sqrt{\pi}^{\frac{n(n-1)}{2}}}=\frac{\sqrt{2}^{m+n} \pi^{m} 2^{m^{2}}}{\prod_{j=0}^{m-1}(j ! \Gamma(j+3 / 2))} .
$$


But

$$
\begin{aligned}
\ln \left(\prod_{j=0}^{m-1}(j ! \Gamma(j+3 / 2))\right) & =2 \sum_{j=1}^{m-1}\left(j \ln j-j+\frac{3}{4} \ln j\right)+O(n) \\
& =\frac{(n-1)^{2}}{4} \ln \left(\frac{n-1}{2}\right)-\frac{3}{8}(n-1)^{2}+\frac{n-1}{4} \ln \left(\frac{n-1}{2}\right)+O(n) .
\end{aligned}
$$

We deduce that

$$
\ln \left(\frac{\operatorname{Vol}\left(O_{n}(\mathbb{R})\right)}{\sqrt{2}^{n} \sqrt{\pi}^{\frac{n(n-1)}{2}}}\right)=\frac{n^{2}}{4} \ln (2)-\frac{n^{2}}{4} \ln (n)+\frac{n}{2} \ln (n)+\frac{n^{2}}{4} \ln 2+\frac{3}{8} n^{2}-\frac{n}{4} \ln n+O(n)
$$

and the result.

\subsection{Determinants of random symmetric matrices}

\subsubsection{Complex symmetric matrices}

For every $n \in \mathbb{N}^{*}$, denote by $S_{n}$ the group of permutations of $\{1, \cdots, n\}$ and for every $\sigma \in S_{n}$, by Cycles $(\sigma)$ the set of cycles appearing in the decomposition of $\sigma$ into a product of cycles with disjoint supports. For instance, if $\sigma$ denotes the permutation $\left(\begin{array}{lllll}1 & 2 & 3 & 4 & 5 \\ 3 & 2 & 1 & 5 & 4\end{array}\right)$ of $\{1, \cdots, 5\}$, then Cycles $(\sigma)=\{(13),(2),(45)\}$.

Lemma 9 For every $n \in \mathbb{N}^{*}, e_{\mathbb{C}}(n)=\sum_{\sigma \in S_{n}} 2^{\# \operatorname{Cycles}(\sigma)}$.

Proof. For every $A \in \operatorname{Sym}(n, \mathbb{C})$, denote by $A=\sum_{1 \leq i \leq j \leq n} a_{i j} \widetilde{E}_{i j}$ and set $a_{j i}=a_{i j}$ if $i>j$. By definition,

$$
\begin{aligned}
& e_{\mathbb{C}}(n)=\int_{\operatorname{Sym}(n, \mathbb{C})}(\operatorname{det} A)(\overline{\operatorname{det} A}) d \mu_{\mathbb{C}}(A) \\
& =\sum_{\sigma \in S_{n}}(-1)^{\epsilon(\sigma)} \sum_{\tau \in S_{n}}(-1)^{\epsilon(\tau)} \int_{S y m(n, \mathbb{C})} \sqrt{2} \# \operatorname{Fix}(\sigma)+\# \operatorname{Fix}(\tau) \ldots \\
& \cdots a_{1 \sigma(1)} \overline{a_{1 \tau(1)}} \cdots a_{n \sigma(n)} \overline{a_{n \tau(n)}} d \mu_{\mathbb{C}}(A),
\end{aligned}
$$

since the diagonal entries of $A$ have weight $\sqrt{2}$. Now, the integral $\int_{\mathbb{C}} z^{\alpha} \bar{z}^{\beta} d \mu_{\mathbb{C}}(z)$ vanishes when $\alpha \neq \beta$, so that for every $\sigma \in S_{n}$, the only permutations $\tau \in S_{n}$ which contribute to the integral are the ones for which $\left\{a_{1 \sigma(1)}, \cdots, a_{n \sigma(n)}\right\}=\left\{a_{1 \tau(1)}, \cdots, a_{n \tau(n)}\right\}$. This implies that for every $j \in\{1, \cdots, n\},\left\{a_{j \sigma(j)}, a_{\sigma^{-1}(j) j}\right\}=\left\{a_{j \tau(j)}, a_{\tau^{-1}(j) j}\right\}$.

If $j$ belongs to a cycle of length 1 or 2 of $\sigma$, we deduce that $\sigma(j)=\tau(j)$. More generally, we deduce that if $\tilde{\sigma}$ is an element of $\operatorname{Cycles}(\sigma)$, then either $\tilde{\sigma}$ or $\tilde{\sigma}^{-1}$ is an element of $\operatorname{Cycles}(\tau)$. In particular, $\epsilon(\sigma)=\epsilon(\tau)$. Conversely, every permutation $\tau$ which can be written as a product of the form $\prod_{\tilde{\sigma} \in \operatorname{Cycles}(\sigma)} \tilde{\sigma}^{ \pm 1}$ contributes to the integral. There are $2^{\# \text { Cycles } \geq 3}(\sigma)$ such permutations, where Cycles ${ }_{\geq 3}(\sigma)$ denotes the set of elements of Cycles $(\sigma)$ having length $\geq 3$. As a consequence,

$$
e_{\mathbb{C}}(n)=\sum_{\sigma \in S_{n}} 2^{\# \text { Cycles }_{\neq 2}(\sigma)} \int_{S y m(n, \mathbb{C})} \prod_{i=1}^{n}\left|a_{i \sigma(i)}\right|^{2} d \mu_{\mathbb{C}}(A)
$$


where $C y \operatorname{cles}_{\neq 2}(\sigma)$ denotes the subset of elements in Cycles $(\sigma)$ having length different from 2. Now, $\int_{\mathbb{C}}|z|^{2} d \mu_{\mathbb{C}}(z)=1$ whereas $\int_{\mathbb{C}}|z|^{4} d \mu_{\mathbb{C}}(z)=2$. Every transposition of Cycles $(\sigma)$ produces an element of this second type whereas the other elements of Cycles $(\sigma)$ give rise to products of the first type. Hence the result.

Lemma 10 For every $n \in \mathbb{N}^{*}, \sum_{\sigma \in S_{n}} 2^{\# \operatorname{Cycles}(\sigma)}=(n+1)$ !

Proof. When $n=1$, this equality is satisfied. Assume that it is satisfied up to a rank $n$ and let us prove it for the rank $n+1$. Let $\sigma \in S_{n+1}$ and $\sigma=\tilde{\sigma}_{1} \cdots \tilde{\sigma}_{k}$ be its decomposition into a product of cycles with disjoint supports. If we remove the element $(n+1)$ of the cycle which contains this element, we get a permutation $\tau$ of $S_{n}$ together with its decomposition into a product of cycles with disjoint supports. We deduce from this a $(n+1)$ to 1 forgetful map $f_{n}: \sigma \in S_{n+1} \mapsto \tau \in S_{n}$ such that $\# \operatorname{Cycles}(\sigma)=\# \operatorname{Cycles}\left(f_{n}(\sigma)\right)$ if $(n+1)$ is not fixed by $\sigma$ and $\# \operatorname{Cycles}(\sigma)=$ $\#$ Cycles $\left(f_{n}(\sigma)\right)+1$ otherwise. Hence,

$$
\sum_{\sigma \in S_{n+1}} 2^{\# \text { Cycles }(\sigma)}=\sum_{\tau \in S_{n}} \sum_{\sigma \in f_{n}^{-1}(\tau)} 2^{\# \text { Cycles }(\sigma)}=(n+2) \sum_{\tau \in S_{n}} 2^{\# \text { Cycles }(\tau)}=(n+2) !
$$

by induction.

Proposition 5 For every $n \in \mathbb{N}, e_{\mathbb{C}}(n)=(n+1)$ !

Proof. This Proposition is a consequence of Lemmas 9 and 10 when $n>0$ and of our convention when $n=0$.

\subsubsection{Real symmetric matrices of odd size}

We recall here the values of $e_{\mathbb{R}}(n), n>0$, distinguishing between the cases $n$ even and $n$ odd, see $\S 25.5$ and $\S 26.6$ of [16. The odd case turns out to be easier than the even one:

Proposition 6 (Formula 26.5.2 of [16]) For every odd integer $n$,

$$
e_{\mathbb{R}}(n)=\frac{2 \sqrt{2}}{\pi} \Gamma\left(\frac{n+2}{2}\right) .
$$

Let us briefly recall the proof of this Proposition [6] as taken out from [16].

Proof. As in the proof of Proposition 4, it follows from the coarea formula (see Theorem 3.2.3 of [10] or Theorem 1 of [21]) that

$$
e_{\mathbb{R}}(n)=\frac{\operatorname{Vol}\left(O_{n}(\mathbb{R})\right)}{2^{n} \sqrt{\pi}^{\frac{n(n-1)}{2}}} \int_{\lambda_{1}<\cdots<\lambda_{n}}\left|\prod_{i=1}^{n} \sqrt{2} \lambda_{i}\right| \prod_{1 \leq i<j \leq n}\left|\lambda_{j}-\lambda_{i}\right| d \mu(\lambda),
$$

where as before the volume of $O_{n}(\mathbb{R})$ is computed with respect to the right invariant metric for which the basis $\left(E_{i j}-E_{j i}\right)_{1 \leq i<j \leq n}$ of its Lie algebra is orthonormal, see 2.1.3, and where $d \mu(\lambda)$ denotes the Gaussian measure on $\mathbb{R}^{n}$. The integrand is a 
Vandermonde determinant. Integrating the odd lines of this determinant and then expanding by pairs of rows in the Laplace manner, we get the relation

$$
e_{\mathbb{R}}(n)=\frac{\operatorname{Vol}\left(O_{n}(\mathbb{R})\right)}{\sqrt{2}^{n} \sqrt{\pi}^{\frac{n(n-1)}{2}}} \operatorname{det} B .
$$

Here, writing $n=2 m+1, B$ denotes a square matrix of size $(m+1) \times(m+1)$ with entries $\left(b_{i j}\right)_{0 \leq i<j \leq m}$ defined by

$$
\forall 0 \leq i \leq m, \forall 0 \leq j<m, b_{i j}=2\left(\psi_{i j}+\eta_{2 i} \eta_{2 j+1}\right)
$$

and $b_{i m}=2 \eta_{2 i}$ where

$$
\psi_{i j}=\int_{0 \leq x<y<+\infty}|x y|\left(x^{2 i} y^{2 j+1}-y^{2 i} x^{2 j+1}\right) d \mu(x) d \mu(y)
$$

and

$$
\eta_{k}=\int_{0}^{+\infty} x^{k+1} d \mu(x)=\frac{1}{2 \sqrt{\pi}} \Gamma\left(\frac{k+2}{2}\right) .
$$

Using linear combinations of rows and columns of $B$ with the help of the relations

$$
\forall i, j \geq 0, \psi_{i+1, j}=(i+1) \psi_{i j}-\frac{1}{\pi 2^{i+j+7 / 2}} \Gamma(i+j+5 / 2)
$$

and $\eta_{2 i+2}=(i+1) \eta_{2 i}$, we get

$$
\begin{aligned}
\operatorname{det} B & =\frac{1}{\sqrt{\pi}^{n} \sqrt{2}^{m} 2^{m(m+1)}} \operatorname{det}(\Gamma(i+j+5 / 2))_{0 \leq i, j \leq m-1} \\
& \left.=\frac{1}{\sqrt{\pi}^{n} \sqrt{2}^{m} 2^{m(m+1)}} \prod_{j=0}^{m-1}(j ! \Gamma(5 / 2+j))(\text { see formula A.18.7 in [16] })\right) .
\end{aligned}
$$

When $n=2 m+1$ we deduce from this that

$$
e_{\mathbb{R}}(n)=\frac{\operatorname{Vol}\left(O_{n}(\mathbb{R})\right)}{\sqrt{\pi}^{\frac{n(n+1)}{2}} \sqrt{2}^{m+1} 2^{m(m+2)}} \prod_{j=0}^{m-1}(j ! \Gamma(5 / 2+j)) .
$$

The result now follows from Lemma 7 .

The proof of Proposition 6 may also provide an alternative proof of Lemma 7 for odd n's, as suggested in [16].

Alternative proof of Lemma 7 in odd dimensions. Proceeding as in the proof of Proposition 6, we get

$$
1=\frac{\operatorname{Vol}\left(O_{n}(\mathbb{R})\right)}{2^{n} \sqrt{\pi}^{\frac{n(n-1)}{2}}} \int_{\lambda_{1}<\cdots<\lambda_{n}} \prod_{1 \leq i<j \leq n}\left(\lambda_{j}-\lambda_{i}\right) d \mu(\lambda)=\frac{\operatorname{Vol}\left(O_{n}(\mathbb{R})\right)}{2^{n} \sqrt{\pi}^{\frac{n(n-1)}{2}}} \operatorname{det}\left(B^{\prime}\right),
$$

where $B^{\prime}$ denotes a square matrix of size $(m+1) \times(m+1)$ with entries $\left(b_{i j}^{\prime}\right)_{0 \leq i, j \leq m}$ defined by

$$
\forall 0 \leq i \leq m, \forall 0 \leq j<m, b_{i j}^{\prime}=2\left(\psi_{i j}^{\prime}+\eta_{2 i-1} \eta_{2 j}\right)
$$


and $b_{i m}^{\prime}=2 \eta_{2 i-1}$, whereas

$$
\psi_{i j}^{\prime}=\int_{0 \leq x<y \leq+\infty}\left(x^{2 i} y^{2 j+1}-y^{2 i} x^{2 j+1}\right) d \mu(x) d \mu(y)=-\psi_{j(i-1)} .
$$

From linear combinations and the relation

$$
\forall i, j \geq 0, \psi_{i+1, j}^{\prime}=\left(\frac{2 i+1}{2}\right) \psi_{i j}^{\prime}-\frac{1}{\pi 2^{i+j+5 / 2}} \Gamma(i+j+3 / 2),
$$

we get

$$
\operatorname{det}\left(B^{\prime}\right)=\frac{1}{\pi^{m} \sqrt{2}^{m} 2^{m^{2}}} \prod_{j=0}^{m-1}(j ! \Gamma(3 / 2+j)) .
$$

Finally,

$$
\operatorname{Vol}\left(O_{n}(\mathbb{R})\right)=\frac{\sqrt{\pi}^{m(n+2)} \sqrt{2}^{m} 2^{m^{2}+n}}{\prod_{j=0}^{m-1}(j ! \Gamma(3 / 2+j))}
$$

and $e_{\mathbb{R}}(n)=\frac{2 \sqrt{2}}{\pi} \Gamma\left(\frac{n+2}{2}\right)$, since $\Gamma(1 / 2)=\sqrt{\pi}$.

Remark 6 The first values given by Proposition 6 are

$$
e_{\mathbb{R}}(1)=\sqrt{\frac{2}{\pi}}, e_{\mathbb{R}}(3)=\frac{3}{\sqrt{2 \pi}} \text { and } e_{\mathbb{R}}(5)=\frac{15}{2 \sqrt{2 \pi}} .
$$

Moreover, from Stirling's formula, $e_{\mathbb{R}}(n)$ is equivalent to $\frac{2 \sqrt{2}}{\pi} \sqrt{m} m$ ! as $n=2 m+1$ grows to infinity.

\subsubsection{Real symmetric matrices of even size}

When the dimension $n=2 m$ is even, the value of $e_{\mathbb{R}}(n)$ is given by the following Proposition 7.

Proposition 7 For every even positive integer $n=2 m$,

$$
e_{\mathbb{R}}(n)=(-1)^{m} \frac{n !}{m ! 2^{n}}+(-1)^{m-1} \frac{4 \sqrt{2} n !}{\sqrt{\pi} m ! 2^{n}} \sum_{k=0}^{m-1}(-1)^{k} \frac{\Gamma(k+3 / 2)}{k !} .
$$

The expression given by Proposition 7 can be rewritten as

$$
e_{\mathbb{R}}(n)=(-1)^{m} \frac{4 \sqrt{2} n !}{\sqrt{\pi} m ! 2^{n}} \int_{0}^{+\infty} \sqrt{t}\left(e^{-t}-\sum_{k=0}^{m-1} \frac{(-t)^{k}}{k !}\right) e^{-t} d t .
$$

The first term in the right-hand side of the expression given by Proposition 7 is alternated and negligible for large values of $n$ with respect to the second one which is always non negative. The latter can be checked by pairing the terms of the sum, see the proof of Corollary 3 . 
Remark 7 The first values of $e_{\mathbb{R}}(n)$ for even n's are:

$$
\begin{array}{lcc}
e_{\mathbb{R}}(0)=1, & e_{\mathbb{R}}(2)=\sqrt{2}-1 / 2, & e_{\mathbb{R}}(4)=\frac{3}{4}(\sqrt{2}+1), \\
e_{\mathbb{R}}(6)=\frac{165}{32} \sqrt{2}-\frac{15}{8}, & e_{\mathbb{R}}(8)=\frac{3 \times 5 \times 7}{16}\left(\frac{13}{8} \sqrt{2}+1\right) . &
\end{array}
$$

Note that $e_{\mathbb{R}}(n)$ is algebraic in $\mathbb{Q}[\sqrt{2}]$ for even $n$ and transcendental for odd values of $n$.

Corollary 3 Whatever the parity of $n$ is, $e_{\mathbb{R}}(n)$ gets equivalent to $\frac{2 \sqrt{2}}{\pi} \Gamma\left(\frac{n+2}{2}\right)$ as $n$ grows to infinity.

Proof. For every odd $n, e_{\mathbb{R}}(n)=\frac{2 \sqrt{2}}{\pi} \Gamma\left(\frac{n+2}{2}\right)$ from Proposition 6. When $n=2 m$ is even, the first term in the right-hand side given by Proposition 7 is equivalent to $(-1)^{m} m^{m} e^{-m} \sqrt{2}$, that is $(-1)^{m} \frac{\Gamma\left(\frac{n+2}{2}\right)}{\sqrt{\pi m}}$ from Stirling's formula. Pairing the terms of the sum in the second one, we get

$$
\sum_{k=0}^{m-1}(-1)^{k} \frac{\Gamma(k+3 / 2)}{k !}=-\frac{1}{2} \sum_{j=0}^{\frac{m}{2}-1} \frac{\Gamma(2 j+3 / 2)}{(2 j+1) !}
$$

when $m$ is even and

$$
\sum_{k=0}^{m-1}(-1)^{k} \frac{\Gamma(k+3 / 2)}{k !}=\frac{\Gamma(m+1 / 2)}{(m-1) !}-\frac{1}{2} \sum_{j=0}^{\frac{m-1}{2}-1} \frac{\Gamma(2 j+3 / 2)}{(2 j+1) !}
$$

when $m$ is odd. In both cases, this sum gets equivalent to $(-1)^{m-1} \frac{\sqrt{m}}{2}$ as $n$ grows to infinity, hence the result.

In order to prove Proposition 17, we first compute in the following Proposition 8 $e_{\mathbb{R}}(n)$ in terms of a sequence $\left(b_{m}\right)_{m \in \mathbb{N}}$ which we now introduce. Let $\left(a_{j}\right)_{j=0}$ be the sequence defined by the relations $a_{0}=\frac{8 \sqrt{2}-7}{3}$ and

$$
\forall j>0, a_{j}=\left(\frac{4 j+2}{2 j+3}\right) a_{j-1}+1 .
$$

Let $b_{1}=a_{0}+1=\frac{4}{3}(2 \sqrt{2}-1)$ and for every $m>1$,

$$
b_{m}=\sum_{j=0}^{m-1}(-1)^{m-1-j}\left(\begin{array}{c}
m-1 \\
j
\end{array}\right) a_{j} .
$$

Proposition 8 For every even integer $n=2 m>0$,

$$
e_{\mathbb{R}}(n)=\frac{n ! \Gamma\left(\frac{n+3}{2}\right) b_{m}}{m !(m-1) ! 2^{n} \sqrt{\pi}}
$$


Proof. As in the proof of Proposition 6, we establish that

$$
e_{\mathbb{R}}(n)=\frac{\operatorname{Vol}\left(O_{n}(\mathbb{R})\right)}{\sqrt{2}^{n} \sqrt{\pi}^{\frac{n(n-1)}{2}}} \operatorname{det}(C),
$$

where $C$ denotes a square matrix of size $m \times m$ and entries $\left(c_{i j}\right)_{0 \leq i, j \leq m-1}$ satisfying

$$
\forall 0 \leq i, j \leq m-1, c_{i j}=2\left(\psi_{i j}+\eta_{2 i} \eta_{2 j+1}\right)
$$

with

$$
\psi_{i j}=\int_{0 \leq x<y \leq+\infty}|x y|\left(x^{2 i} y^{2 j+1}-y^{2 i} x^{2 j+1}\right) d \mu(x) d \mu(y)
$$

and $\eta_{k}=\int_{0}^{+\infty} x^{k+1} d \mu(x)=\frac{1}{2 \sqrt{\pi}} \Gamma\left(\frac{k+2}{2}\right)$. Following $\S 26.6$ of [16],

$$
\begin{aligned}
& \operatorname{det}(C)=-2^{m}\left|\begin{array}{cc}
-1 & \left(\eta_{2 j+1}\right)_{0 \leq j \leq m-1} \\
\left(\eta_{2 i}\right)_{0 \leq i \leq m-1} & \left(\psi_{i j}\right)_{0 \leq i, j \leq m-1}
\end{array}\right| \\
& =-2^{m}\left|\begin{array}{cc}
-1 & \left(\eta_{2 j+1}\right)_{0 \leq j \leq m-1} \\
\eta_{0} & \left(\psi_{0 j}\right)_{0 \leq j \leq m-1} \\
0 & \left(\psi_{i j}-i \psi_{i-1 j}=-\frac{\Gamma(i+j+3 / 2)}{\pi 2^{i+j+5 / 2}}\right)_{1 \leq i \leq m-1,0 \leq j \leq m-1}
\end{array}\right| \\
& =\frac{(-1)^{m}}{\pi^{m} 2^{m^{2}} \sqrt{2}^{m-1}}\left|\begin{array}{ccc}
-1 & 0 & \\
2 \sqrt{\pi} & & 2^{j}\left(4 \pi \psi_{0 j}+\Gamma(j+3 / 2)\right)_{0 \leq j \leq m-1} \\
0 & \Gamma(5 / 2) & \Gamma(j+5 / 2)_{1 \leq j \leq m-1} \\
0 & 0 & (\Gamma(i+j+3 / 2)-(i+1 / 2) \Gamma(i+j+1 / 2) \\
& & =j \Gamma(i+j+1 / 2))_{2 \leq i \leq m-1,1 \leq j \leq m-1}
\end{array}\right|,
\end{aligned}
$$

which equals

$$
\frac{(-1)^{m-1} \prod_{j=0}^{m-1}(j ! \Gamma(j+5 / 2))}{\pi^{m} 2^{m^{2}} \sqrt{2}^{m-1}}\left|\begin{array}{cccc}
\frac{2^{j}}{j ! \Gamma(j+5 / 2)} & & & \left(4 \pi \psi_{0 j}+\Gamma(j+3 / 2)\right)_{0 \leq j \leq m-1} \\
1 & & & (1 / j !)_{1 \leq j \leq m-1} \\
0 & 1 & & (1 /(j-1) !)_{2 \leq j \leq m-1} \\
\vdots & \ddots & \ddots & \vdots \\
0 & \ldots & 0 & 1
\end{array}\right|
$$

so that the entry of the $i$-th row and $j$-th column, $1 \leq i \leq m-1,0 \leq j \leq m-1$ equals $1 /(j-i+1)$ ! if $j-i+1 \geq 0$ and 0 otherwise. Subtracting to the $m-1$ first lines multiples of the last one, we obtain zeros on the last column, whereas the entry of the penultimate column on the $i-$ th line, $1 \leq i \leq m-2$ equals $\frac{m-1-i}{(m-i) !}$. Then, subtracting to the $m-2$ first lines multiples of the penultimate one, we get zeros on the penultimate column whereas the entry of the $(m-2)$-th column on the $i$-th line, $1 \leq i \leq m-3$, equals $\frac{(m-1-i)(m-2-i)}{(m-i) !}$. By recurrence, we get a lower triangular matrix and




with

$$
\alpha=\sum_{j=0}^{m-1}(-1)^{j} \frac{2^{j} \prod_{k=m-j}^{m-1} k}{j ! \Gamma(j+5 / 2)}\left(4 \pi \psi_{0 j}+\Gamma(j+3 / 2)\right) .
$$

We deduce then from Lemma 7 the relation

$$
e_{\mathbb{R}}(n)=\frac{n ! \Gamma\left(\frac{n+3}{2}\right)}{m !(m-1) ! 2^{n} \sqrt{\pi}} 2 \sqrt{2} \sum_{j=0}^{m-1}(-1)^{m-1-j} \frac{2^{j}\left(\begin{array}{c}
m-1 \\
j
\end{array}\right)}{\Gamma(j+5 / 2)}\left(4 \pi \psi_{0 j}+\Gamma(j+3 / 2)\right) .
$$

The result follows by setting, for every $j \in\{0, \cdots, m-1\}$,

$$
a_{j}=\frac{2^{j+1} \sqrt{2}}{\Gamma(j+5 / 2)}\left(4 \pi \psi_{0 j}+\Gamma(j+3 / 2)\right)-1 .
$$

Indeed,

$$
\psi_{00}=\frac{1}{8 \sqrt{2 \pi}}(\sqrt{2}-1)
$$

so that $a_{0}=\frac{8 \sqrt{2}-7}{3}$ and the recurrence relation satisfied by $\left(a_{j}\right)_{j \geq 0}$ follows from the relation

$$
\forall j>0, \psi_{0 j}=(j+1 / 2) \psi_{0 j-1}+\frac{1}{\pi 2^{j+5 / 2}} \Gamma(j+3 / 2)
$$

(compare formula 26.4.13 of [16]).

Remark 8 As in the alternative proof of Lemma 7 , we may get that for every $n=$ $2 m>0$,

$$
\operatorname{Vol}\left(O_{n}(\mathbb{R})\right)=\frac{2^{m(m+1)} \sqrt{2}^{m} \sqrt{\pi}^{\frac{n(n+1)}{2}}(m-1) !}{\prod_{j=0}^{m-1}(j ! \Gamma(j+3 / 2)) b_{m}^{\prime}},
$$

where $b_{1}^{\prime}=a_{0}^{\prime}+1$ and for every $m>1, b_{m}^{\prime}=\sum_{j=0}^{m-1}(-1)^{m-1-j}\left(\begin{array}{c}m-1 \\ j\end{array}\right) a_{j}^{\prime}$. This sequence $\left(a_{j}^{\prime}\right)_{j \geq 0}$ is defined by the relations $a_{0}^{\prime}=1$ and $\forall j>0, a_{j}=\left(\frac{4 j}{2 j+1}\right) a_{j-1}+1$.

Proof of Proposition 7. When $m=1$, Proposition 7 is a consequence of Proposition 8 since $b_{1}=\frac{4}{3}(2 \sqrt{2}-1)$. From Proposition 8 , when $m>1$, we have to compute the values of $b_{m} \in \mathbb{Q}[\sqrt{2}]$. For every $j \geq 0$, we deduce from the recurrence relation that

$$
a_{j}=\frac{2^{j}}{2 j+3}\left(8 \sqrt{2}-7+2 \sum_{k=2}^{j} \frac{2 k+1}{2^{k}}\right)+1 .
$$

Writing $\sum_{k=0}^{j} \frac{2 k+1}{2^{k}}=\left(\sum_{k=0}^{j} \frac{x^{2 k+1}}{2^{k}}\right)_{\mid x=1}^{\prime}=6-\frac{2 j+5}{2^{j}}$, we deduce

$$
\forall j>0, a_{j}=8 \sqrt{2} \frac{2^{j}}{2 j+3}-\frac{4}{2 j+3}-1
$$

and as a consequence

$$
b_{m}=4 \sum_{j=0}^{m-1}(-1)^{m-1-j}\left(\begin{array}{c}
m-1 \\
j
\end{array}\right) \frac{2 \sqrt{2} 2^{j}-1}{2 j+3} .
$$


Now,

$$
\begin{aligned}
\left(\sum_{j=0}^{m-1}(-1)^{m-1-j}\left(\begin{array}{c}
m-1 \\
j
\end{array}\right) \frac{2 \sqrt{2} 2^{j}-1}{2 j+3} x^{2 j+3}\right)^{\prime} & =\sum_{j=0}^{m-1}(-1)^{m-1-j}\left(\begin{array}{c}
m-1 \\
j
\end{array}\right)\left(2 \sqrt{2} 2^{j}-1\right) x^{2 j+2} \\
& =x^{2}\left(2 \sqrt{2}\left(2 x^{2}-1\right)^{m-1}-\left(x^{2}-1\right)^{m-1}\right),
\end{aligned}
$$

so that

$$
b_{m}=8 \sqrt{2} \int_{0}^{1} x^{2}\left(2 x^{2}-1\right)^{m-1} d x-4 \int_{0}^{1} x^{2}\left(x^{2}-1\right)^{m-1} d x .
$$

From the relations

$$
\begin{aligned}
\forall j>0 \quad \int_{0}^{1} x^{2}\left(x^{2}-1\right)^{j} d x & =\frac{-2 j}{2 j+3} \int_{0}^{1} x^{2}\left(x^{2}-1\right)^{j-1} \text { and } \\
\int_{0}^{1} x^{2}\left(2 x^{2}-1\right)^{j} d x & =\frac{-2 j}{2 j+3} \int_{0}^{1} x^{2}\left(2 x^{2}-1\right)^{j-1}+\frac{1}{2 j+3},
\end{aligned}
$$

follows that

$$
b_{m}=(-1)^{m-1} \frac{4 \sqrt{2}(m-1) !}{\Gamma(m+3 / 2)} \sum_{k=0}^{m-1}(-1)^{k} \frac{\Gamma(k+3 / 2)}{k !}+(-1)^{m} \frac{(m-1) ! \sqrt{\pi}}{\Gamma(m+3 / 2)} .
$$

Now, Proposition 7 follows from Proposition 8 .

Remark 9 An expression of $e_{\mathbb{R}}(n)$ in terms of hypergeometric series can be extracted from [7], whereas an equivalent in logarithmic scale can be extracted from [22].

\subsubsection{Values of $e(p, q)$ for $p+q \leq 3$}

We have not been able to compute the numbers $e_{\mathbb{R}}(p, q)$ in general and only give here their values for $p+q \leq 3$.

\section{Lemma 11}

$$
\begin{aligned}
e_{\mathbb{R}}(1,0)=e_{\mathbb{R}}(0,1) & =\frac{1}{\sqrt{2 \pi}}, \\
e_{\mathbb{R}}(2,0)=e_{\mathbb{R}}(0,2) & =\frac{1}{4}(\sqrt{2}-1), e_{\mathbb{R}}(1,1)=\frac{1}{\sqrt{2}} \text { and } \\
\forall p \in\{0, \cdots, 3\}, e_{\mathbb{R}}(p, 3-p) & =\frac{3}{4 \sqrt{2 \pi}}-\frac{(-1)^{p}}{2 \sqrt{\pi}} .
\end{aligned}
$$

Proof. The multiplication of symmetric matrices by -Id preserves the measure of $\operatorname{Sym}(n, \mathbb{R})$ as well as the absolute value of the determinant, so that for every $p, q \in \mathbb{N}$, $e_{\mathbb{R}}(p, q)=e_{\mathbb{R}}(q, p)$. As a consequence, $e_{\mathbb{R}}(1,0)=e_{\mathbb{R}}(0,1)=\frac{1}{2} e_{\mathbb{R}}(1)=\frac{1}{\sqrt{2 \pi}}$ from Proposition 6, since $\Gamma(3 / 2)=\frac{\sqrt{\pi}}{2}$. Proceeding as in the beginning of the proof of Proposition 6, we get that

$$
\begin{aligned}
e_{\mathbb{R}}(2,0) & =\frac{\operatorname{Vol}\left(O_{2}(\mathbb{R})\right)}{2 \sqrt{\pi}} \int_{0<\lambda_{1}<\lambda_{2}<+\infty} \operatorname{det}\left(\begin{array}{cc}
\left|\lambda_{1}\right| & \lambda_{1}^{2} \\
\left|\lambda_{2}\right| & \lambda_{2}^{2}
\end{array}\right) d \mu\left(\lambda_{1}\right) d \mu\left(\lambda_{2}\right) \\
& =2 \sqrt{2 \pi} \psi_{00},
\end{aligned}
$$


by (6) and Lemma 7 up to which $\operatorname{Vol}\left(O_{2}(\mathbb{R})\right)=4 \pi \sqrt{2}$. But $\psi_{00}=\frac{1}{8 \sqrt{2 \pi}}(\sqrt{2}-1)$ by (8)), so that $e_{\mathbb{R}}(2,0)=e_{\mathbb{R}}(0,2)=\frac{1}{4}(\sqrt{2}-1)$. Likewise,

$$
\begin{aligned}
& e_{\mathbb{R}}(1,1)=\frac{\operatorname{Vol}\left(O_{2}(\mathbb{R})\right)}{2 \sqrt{\pi}} \int_{-\infty}^{0} \int_{0}^{+\infty} \operatorname{det}\left(\begin{array}{ll}
\left|\lambda_{1}\right| & \lambda_{1}^{2} \\
\left|\lambda_{2}\right| & \lambda_{2}^{2}
\end{array}\right) d \mu\left(\lambda_{1}\right) d \mu\left(\lambda_{2}\right) \\
& =4 \sqrt{2 \pi} \eta_{0} \eta_{1} \text { from (6) } \\
& =\frac{1}{\sqrt{2}} \text {. }
\end{aligned}
$$

We get along the same lines that

$$
e_{\mathbb{R}}(3,0)=\frac{\operatorname{Vol}\left(O_{3}(\mathbb{R})\right)}{\sqrt{2 \pi}^{3}}\left(\eta_{2} \psi_{00}-\eta_{0} \psi_{10}-\eta_{1} \int_{0 \leq x<y<+\infty}|x y|\left(y^{2}-x^{2}\right) d \mu(x) d \mu(y)\right) .
$$

From (7), $\operatorname{Vol}\left(\mathrm{O}_{3}(\mathbb{R})\right)=2^{5} \sqrt{2} \pi^{2}$. From the recurrence relation given in the proof of Proposition 6, we deduce that $\psi_{10}=\frac{1}{8 \sqrt{\pi}}-\frac{7 \sqrt{2}}{64 \sqrt{\pi}}$. Finally, $\forall i \geq 0, \forall j>0$,

$$
\begin{aligned}
\int_{0 \leq x<y<+\infty}|x y|\left(x^{2 i} y^{2 j}-x^{2 j} y^{2 i}\right) d \mu(x) d \mu(y)= & j \int_{0 \leq x<y<+\infty}|x y|\left(x^{2 i} y^{2 j-2}-x^{2 j-2} y^{2 i}\right) d \mu(x) d \mu(y) \\
& +\frac{(i+j) !}{\pi 2^{i+j+2}}
\end{aligned}
$$

so that $\int_{0 \leq x<y<+\infty}|x y|\left(y^{2}-x^{2}\right) d \mu(x) d \mu(y)=\frac{1}{8 \pi}$. It follows that

$$
\begin{aligned}
e_{\mathbb{R}}(3,0)=e_{\mathbb{R}}(0,3) & =16 \sqrt{\pi}\left(\frac{(\sqrt{2}-1)}{16 \sqrt{2} \pi}-\frac{1}{16 \pi}+\frac{7}{64 \sqrt{2} \pi}-\frac{1}{32 \pi}\right) \\
& =-\frac{1}{2 \sqrt{\pi}}+\frac{3}{4 \sqrt{2 \pi}} .
\end{aligned}
$$

Likewise,

$$
\begin{aligned}
e_{\mathbb{R}}(2,1)=e_{\mathbb{R}}(1,2) & =\frac{\operatorname{Vol}\left(O_{3}(\mathbb{R})\right)}{\sqrt{2 \pi}^{3}}\left(-\eta_{0} \psi_{10}+\eta_{2} \psi_{00}+\eta_{1} \int_{0 \leq x<y<+\infty}|x y|\left(y^{2}-x^{2}\right) d \mu(x) d \mu(y)\right) \\
& =16 \sqrt{\pi}\left(\frac{(\sqrt{2}-1)}{16 \sqrt{2} \pi}-\frac{1}{16 \pi}+\frac{7}{64 \sqrt{2} \pi}+\frac{1}{32 \pi}\right) \\
& =\frac{1}{2 \sqrt{\pi}}+\frac{3}{4 \sqrt{2 \pi}} .
\end{aligned}
$$

\section{References}

[1] Greg W. Anderson, Alice Guionnet, and Ofer Zeitouni, An introduction to random matrices, Cambridge Studies in Advanced Mathematics, vol. 118, Cambridge University Press, Cambridge, 2010.

[2] Antonio Auffinger, Gérard Ben Arous, and Jiri Černy, Random matrices and complexity of spin glasses, arXiv:1003.1129v2 (2010). 
[3] G. Ben Arous and A. Guionnet, Large deviations for Wigner's law and Voiculescu's non-commutative entropy, Probab. Theory Related Fields 108 (1997), no. 4, 517-542.

[4] Peter Bürgisser, Average Euler characteristic of random real algebraic varieties, C. R. Math. Acad. Sci. Paris 345 (2007), no. 9, 507-512.

[5] David S. Dean and Satya N. Majumdar, Large deviations of extreme eigenvalues of random matrices, Phys. Rev. Lett. 97 (2006), no. 16, 160201, 4.

[6] Jean-Pierre Dedieu and Gregorio Malajovich, On the number of minima of a random polynomial, J. Complexity 24 (2008), no. 2, 89-108.

[7] R. Delannay and G. Le Caër, Distribution of the determinant of a random realsymmetric matrix from the Gaussian orthogonal ensemble, Phys. Rev. E (3) 62 (2000), no. 2, part A, 1526-1536.

[8] Michael R. Douglas, Bernard Shiffman, and Steve Zelditch, Critical points and supersymmetric vacua. I., Commun. Math. Phys. 252 (2004), no. 1-3, 325-358.

[9] _ Critical points and supersymmetric vacua. II: Asymptotics and extremal metrics., J. Differ. Geom. 72 (2006), no. 3, 381-427.

[10] Herbert Federer, Geometric measure theory, Die Grundlehren der mathematischen Wissenschaften, Band 153, Springer-Verlag New York Inc., New York, 1969.

[11] Damien Gayet and Jean-Yves Welschinger, Exponential rarefaction of real curves with many components, Publ. Math. Inst. Hautes Études Sci. (2011), no. 113, 69-96.

[12] What is the total Betti number of a random real hypersurface?, arXiv:1107.2288, to appear in J. Reine Angew. Math. (2012).

[13] Lars Hörmander, Notions of convexity, Modern Birkhäuser Classics, Birkhäuser Boston Inc., Boston, MA, 2007, Reprint of the 1994 edition.

[14] E. Kostlan, On the distribution of roots of random polynomials, From Topology to Computation: Proceedings of the Smalefest (Berkeley, CA, 1990), Springer, New York, 1993, pp. 419-431.

[15] Brian Macdonald, Density of complex critical points of a real random $\mathrm{SO}(m+1)$ polynomial, J. Stat. Phys. 141 (2010), no. 3, 517-531.

[16] Madan Lal Mehta, Random matrices, third ed., Pure and Applied Mathematics (Amsterdam), vol. 142, Elsevier/Academic Press, Amsterdam, 2004.

[17] J. Milnor, Morse theory, Based on lecture notes by M. Spivak and R. Wells. Annals of Mathematics Studies, No. 51, Princeton University Press, Princeton, N.J., 1963.

[18] Fedor Nazarov and Mikhail Sodin, On the number of nodal domains of random spherical harmonics, Amer. J. Math. 131 (2009), no. 5, 1337-1357. 
[19] S. S. Podkorytov, The mean value of the Euler characteristic of an algebraic hypersurface, Algebra i Analiz 11 (1999), no. 5, 185-193.

[20] Bernard Shiffman and Steve Zelditch, Equilibrium distribution of zeros of random polynomials, Int. Math. Res. Not. (2003), no. 1, 25-49.

[21] M. Shub and S. Smale, Complexity of Bezout's theorem. II. Volumes and probabilities, Computational algebraic geometry (Nice, 1992), Progr. Math., vol. 109, 1993, pp. 267-285.

[22] Terence Tao and Van $\mathrm{Vu}$, A central limit theorem for the determinant of a Wigner matrix, arXiv:1111.6300 (2011).

[23] Gang Tian, On a set of polarized Kähler metrics on algebraic manifolds, J. Differential Geom. 32 (1990), no. 1, 99-130.

UNIVERSITÉ DE LYON

CNRS UMR 5208

UNIVERSITÉ LYON 1

Institut CAmille JoRdan

43 blvd. du 11 novembre 1918

F-69622 Villeurbanne cedex

France

gayet@math.univ-lyon1.fr

welschinger@math.univ-lyon1.fr 